\title{
Skew PBW extensions over symmetric rings
}

\section{A. Reyes* and H. Suárez}

\author{
Communicated by V. A. Artamonov
}

\section{Dedicated to the memory of Professor V. A. Artamonov}

\begin{abstract}
Our purpose in this paper is to characterize skew PBW extensions over several weak symmetric rings. As a consequence of our treatment, we extend results in the literature concerning the property of symmetry for commutative rings and skew polynomial rings.
\end{abstract}

\section{Introduction}

A ring $R$ is said to be Armendariz (the term was introduced by Rege and Chhawchharia [43]) if whenever polynomials $f(x)=\sum_{i=0}^{s} a_{i} x^{i}$ and $g(x)=\sum_{j=0}^{t} b_{j} x^{j}$ in $R[x]$ (the commutative polynomial ring in the indeterminate $x$ with coefficients in $R$ ) satisfy $f(x) g(x)=0$, then $a_{i} b_{j}=0$, for all $i, j$. In the context of the well-known Ore extensions (also called skew polynomial rings) introduced by Ore [41], for an endomorphism $\sigma$ and a $\sigma$-derivation $\delta$ of $R$, Moussavi and Hashemi [38] defined $R$ to be $(\sigma, \delta)$-skew Armendariz if for $f(x)=\sum_{i=0}^{s} a_{i} x^{i}$ and $g(x)=\sum_{j=0}^{t} b_{j} x^{j}$ in $R[x ; \sigma, \delta]$ satisfying $f(x) g(x)=0$, then $a_{i} x^{i} b_{j} x^{j}=0$, for each $i, j$.

Of interest for this paper, a ring $R$ is called (i) reduced, if $a^{2}=0$ implies $a=0$, for all $a \in R$; (ii) (Lambek [26]) symmetric, if $a b c=0$ then $a c b=0$, for all $a, b, c, \in R$; (iii) reversible, if $a b=0$ implies $b a=0$, for all

${ }^{*}$ Supported by the research fund of Department of Mathematics, Faculty of Science, Universidad Nacional de Colombia - Sede Bogotá, Colombia, HERMES CODE 52464.

2020 MSC: Primary 16S36, 16S38; Secondary 14A22, 16T20.

Key words and phrases: symmetric ring, noncommutative ring, skew PBW extension. 
$a, b \in R$; (iv) semicommutative, if $a b=0$ then $a R b=0$, for all $a, b \in R$ (Bell [7] defined the following: a ring $R$ is said to satisfy the IFP, insertion of factors property, if $r_{R}(a)$, the right annihilator of $a$ in $R$, is an ideal for all $a \in R$. Sometimes, a semicommutative ring is also called a ring with IFP property). It is known that the implications reduced $\Rightarrow$ symmetric $\Rightarrow$ reversible $\Rightarrow$ semicommutative hold, but, in general, the converse of each one of these implications is false (see Marks [36] for a detailed discussion).

Of course, commutative rings are symmetric. Reduced rings are symmetric as we can appreciate in Anderson and Camillo [2]. Nevertheless, there are many nonreduced commutative (so symmetric) rings. Now, if $R$ is Armendariz, then the ring $R[x]$ over $R$ is symmetric if and only if $R$ is symmetric (Huh et al. [20] and Kim and Lee [24]). In the noncommutative case, there are results concerning this property over $(\sigma, \delta)$-skew Armendariz rings (see Ouyang and Chen [42]). There, the authors defined weak symmetric rings and weak $(\sigma, \delta)$-symmetric rings for the context of Ore extensions $R[x ; \sigma, \delta]$, where $R$ is an associative ring with identity. They proved that for every $(\sigma, \delta)$-compatible and reversible ring $R$ (following Annin [3], for an endomorphism $\sigma$ and a $\sigma$-derivation $\delta$ of $R, R$ is called $\sigma$-compatible, if for every $a, b \in R$, we have $a b=0$ if and only if $a \sigma(b)=0$ necessarily the endomorphism $\sigma$ is injective -, and $R$ is called $\delta$-compatible, if for each $a, b \in R, a b=0$ implies $a \delta(b)=0$. If $R$ is both $\sigma$-compatible and $\delta$-compatible, $R$ is called $(\sigma, \delta)$-compatible $), R$ is weak symmetric if and only if $R[x ; \sigma, \delta]$ is weak symmetric, and for every semi-commutative ring $R, R$ is weak $(\sigma, \delta)$-symmetric if and only if $R[x]$ is weak $(\bar{\sigma}, \bar{\delta})$-symmetric, where $\bar{\sigma}$ and $\bar{\delta}$ are the extended maps of $\sigma$ and $\delta$ over $R[x]$, respectively.

Having in mind all above results and with the aim of establishing more general results about the symmetry property in the context of noncommutative rings more general than (iterated) Ore extensions of injective type (i.e., when $\sigma$ is an injective endomorphism of $R$ ), in this paper we are interested in the skew Poincaré-Birkhoff-Witt $(P B W)$ extensions introduced by Gallego and Lezama [13] with the aim of generalizing the PBW extensions defined by Bell and Goodearl [8]. In section 1, we will say some words about these objects. For the moment, we describe the structure of the article.

The paper is organized as follows. In section 1, we recall some results about skew PBW extensions which will be useful in the rest of the paper. In section 2 , we consider the notions of $\Sigma$-rigid rings and $(\Sigma, \Delta)$-compatible rings which are key throughout the article. Next, in section 3, we present some results about nilpotent elements of skew PBW extensions, and then characterize these extensions over weak symmetric rings. In section 4, we 
investigate skew PBW extensions over weak $(\Sigma, \Delta)$-symmetric rings. The results presented in sections 3 and 4 generalize those presented by Ouyang and Chen [42] for Ore extensions of injective type. Finally, section 5 presents examples where the results obtained in sections 3 and 4 can be illustrated.

Throughout the paper, the word ring means an associative ring (not necessarily commutative) with identity. The letters $k$ and $\mathbb{k}$ will denote a commutative ring and a field, respectively. $\mathbb{N}$ denotes the set of natural numbers including 0 , and $\mathbb{C}$ denotes the set of complex numbers. Finally, for a ring $R, \operatorname{nil}(R)$ represents the set of nilpotent elements of $R$.

\section{Skew PBW extensions}

As we said in the Introduction, skew PBW extensions were defined by Gallego and Lezama [13] with the aim of extending the PBW extensions introduced in [8] (which include the classical commutative polynomial rings, universal enveloping algebra of a Lie algebra, and others). Ore extensions of injective type are strictly contained in skew PBW extensions (this is not possible for original PBW extensions; see [32], for a list of noncommutative rings which are skew PBW extensions but not iterated Ore extensions). Several ring and module theoretical properties of skew PBW extensions have been established by different people (e.g., Acosta [1], Artamonov [4], Hamidizadeh et al. [15], Hashemi et al. [17], [18], [19], Lezama et al. [14], [22], [27], [28], [29], [30], [31], [32], [33], Louzari [35], Niño et al. [39], [40], Tumwesigye et al. [54], Zambrano [55], and the authors [49], [50], [52], [53]). A book containing research results about these extensions has recently been published (see Fajardo et al. [11]).

Skew PBW extensions also generalize another families of noncommutative rings introduced in the literature, and share remarkable examples with another classes of noncommutative algebras . Let us mention briefly some of these algebras (see Fajardo et al. [11] for a detailed reference of each one of these families): (i) universal enveloping algebras of finite dimensional Lie algebras; (ii) PBW extensions introduced by Bell and Goodearl [8]; (iii) almost normalizing extensions defined by McConnell and Robson [37]; (iv) solvable polynomial rings introduced by KandriRody and Weispfenninig [23]; (v) diffusion algebras defined by Isaev et al. [21]; (vi) 3-dimensional skew polynomial algebras defined by Bell and Smith, and studied by Rosenberg [51] (cf. [46]); (vii) the kind of Ore extensions studied by Artamonov et al. [5]; (viii) some deformations of algebras appearing in mathematical physics (cf. Gavrilik and Klimik [12]). 
The advantage of skew PBW extensions is that they do not require the coefficients to commute with the variables and, moreover, the coefficients need not come from a field (see Definition 1). In fact, the skew PBW extensions share well-known groups of algebras such as some types of $G$-algebras introduced by Apel [6] and some PBW algebras defined by Bueso et al. [10] (both $G$-algebras and PBW algebras take coefficients in fields and assume that coefficientes commute with variables), AuslanderGorenstein rings, some Calabi-Yau and skew Calabi-Yau algebras, some Artin-Schelter regular algebras, some Koszul and augmented Koszul algebras, quantum polynomials, some quantum universal enveloping algebras, and others (see Suárez et al. [52] and [53] for more details).

As we can appreciate, skew PBW extensions include several examples of noncommutative rings, which means that a theory of symmetry for these extensions will cover several treatments in the literature and will establish similar results for algebras not considered before. Formulate this theory is the objective of the present paper.

Next, we recall some results about skew PBW extensions which are useful for the rest of the paper.

Definition 1 ([13], Definition 1). Let $R$ and $A$ be rings. We say that $A$ is a skew $P B W$ extension (also known as $\sigma-P B W$ extension) over $R$, which is denoted by $A:=\sigma(R)\left\langle x_{1}, \ldots, x_{n}\right\rangle$, if the following conditions hold:

(i) $R$ is a subring of $A$ sharing the same multiplicative identity element.

(ii) there exist elements $x_{1}, \ldots, x_{n} \in A$ such that $A$ is a left free $R$ module, with basis

$$
\operatorname{Mon}(A):=\left\{x^{\alpha}=x_{1}^{\alpha_{1}} \cdots x_{n}^{\alpha_{n}} \mid \alpha=\left(\alpha_{1}, \ldots, \alpha_{n}\right) \in \mathbb{N}^{n}\right\}
$$

and $x_{1}^{0} \cdots x_{n}^{0}:=1 \in \operatorname{Mon}(A)$.

(iii) For each $1 \leqslant i \leqslant n$ and any $r \in R \backslash\{0\}$, there exists an element $c_{i, r} \in R \backslash\{0\}$ such that $x_{i} r-c_{i, r} x_{i} \in R$.

(iv) For any elements $1 \leqslant i, j \leqslant n$, there exists $d_{i, j} \in R \backslash\{0\}$ such that $x_{j} x_{i}-d_{i, j} x_{i} x_{j} \in R+R x_{1}+\cdots+R x_{n}$, i.e., there exist elements $r_{0}^{(i, j)}, r_{1}^{(i, j)}, \ldots, r_{n}^{(i, j)} \in R$ with $x_{j} x_{i}-d_{i, j} x_{i} x_{j}=r_{0}^{(i, j)}+\sum_{k=1}^{n} r_{k}^{(i, j)} x_{k}$.

Since $\operatorname{Mon}(A)$ is a left $R$-basis of $A$, the elements $c_{i, r}$ and $d_{i, j}$ are unique, ([13], Remark 2).

Proposition 1 ([13], Proposition 3). Let $A$ be a skew PBW extension over $R$. For each $1 \leqslant i \leqslant n$, there exist an injective endomorphism $\sigma_{i}: R \rightarrow R$ and an $\sigma_{i}$-derivation $\delta_{i}: R \rightarrow R$ such that $x_{i} r=\sigma_{i}(r) x_{i}+\delta_{i}(r)$, 
for each $r \in R$. From now on, we will write $\Sigma:=\left\{\sigma_{1}, \ldots, \sigma_{n}\right\}$, and $\Delta:=\left\{\delta_{1}, \ldots, \delta_{n}\right\}$.

Remark 1 ([13], section 3). Let $A=\sigma(R)\left\langle x_{1}, \ldots, x_{n}\right\rangle$ be a skew PBW extension over $R$.

(i) Consider the families $\Sigma$ and $\Delta$ in Proposition 1. Throughout the paper, for any element $\alpha=\left(\alpha_{1}, \ldots, \alpha_{n}\right) \in \mathbb{N}^{n}$, we will write $\sigma^{\alpha}:=$ $\sigma_{1}^{\alpha_{1}} \circ \cdots \circ \sigma_{n}^{\alpha_{n}}, \delta^{\alpha}=\delta_{1}^{\alpha_{1}} \circ \cdots \circ \delta_{n}^{\alpha_{n}}$, where $\circ$ denotes composition, and $|\alpha|:=$ $\alpha_{1}+\cdots+\alpha_{n}$. If $\beta=\left(\beta_{1}, \ldots, \beta_{n}\right) \in \mathbb{N}^{n}$, then $\alpha+\beta:=\left(\alpha_{1}+\beta_{1}, \ldots, \alpha_{n}+\beta_{n}\right)$.

(ii) Given the importance of monomial orders in the proofs of the results presented in section 3, next we recall some key facts about these for skew PBW extensions.

Let $\succeq$ be a total order defined on $\operatorname{Mon}(A)$. If $x^{\alpha} \succeq x^{\beta}$ but $x^{\alpha} \neq x^{\beta}$, we will write $x^{\alpha} \succ x^{\beta}$. If $f$ is a nonzero element of $A$, then $f$ can be expressed uniquely as $f=a_{0}+a_{1} X_{1}+\cdots+a_{m} X_{m}$, with $a_{i} \in R, X_{i}=x^{\alpha_{l}}=$ $x_{1}^{\alpha_{i 1}} \cdots x_{n}^{\alpha_{i n}}$, and $X_{m} \succ \cdots \succ X_{1}$ (eventually, we will use expressions as $f=a_{0}+a_{1} Y_{1}+\cdots+a_{m} Y_{m}$, with $a_{i} \in R$, and $\left.Y_{m} \succ \cdots \succ Y_{1}\right)$. With this notation, we define $\operatorname{lm}(f):=X_{m}$, the leading monomial of $f$; $\operatorname{lc}(f):=a_{m}$, the leading coefficient of $f ; \operatorname{lt}(f):=a_{m} X_{m}$, the leading term of $f ; \exp (f):=\exp \left(X_{m}\right)=\alpha_{m}$, the order of $f$. Note that $\operatorname{deg}(f):=$ $\max \left\{\operatorname{deg}\left(X_{i}\right)\right\}_{i=1}^{m}$. Finally, if $f=0$, then $\operatorname{lm}(0):=0, \operatorname{lc}(0):=0, \operatorname{lt}(0):=0$. We also consider $X \succ 0$ for any $X \in \operatorname{Mon}(A)$. Thus, we extend $\succeq$ to $\operatorname{Mon}(A) \cup\{0\}$.

Following Gallego and Lezama, [13], Definition 11, if $\succeq$ is a total order on $\operatorname{Mon}(A)$, we say that $\succeq$ is a monomial order on $\operatorname{Mon}(A)$ if the following conditions hold:

- For every $x^{\beta}, x^{\alpha}, x^{\gamma}, x^{\lambda} \in \operatorname{Mon}(A)$, we have the implication $x^{\beta} \succeq$ $x^{\alpha} \Rightarrow \operatorname{lm}\left(x^{\gamma} x^{\beta} x^{\lambda}\right) \succeq \operatorname{lm}\left(x^{\gamma} x^{\alpha} x^{\lambda}\right)$ (the total order is compatible with multiplication).

- $x^{\alpha} \succeq 1$, for every $x^{\alpha} \in \operatorname{Mon}(A)$.

- $\succeq$ is degree compatible, i.e., $|\beta| \succeq|\alpha| \Rightarrow x^{\beta} \succeq x^{\alpha}$.

Monomial orders are also called admissible orders. The condition (iii) of the previous definition is needed in the proof of the fact that every monomial order on $\operatorname{Mon}(A)$ is a well order, that is, there are not infinite decreasing chains in $\operatorname{Mon}(A)$ (see [13], Proposition 12). The importance of considering monomial orders on $\operatorname{Mon}(A)$ can be appreciated in Lezama et al. [13] and [22] where the Gröbner theory for left ideals and left modules of skew PBW extensions was studied.

The following result is similar to the established by Bueso et al. [10], Proposition 2.4, for PBW rings. 
Proposition 2 ([13], Theorem 7). If $A$ is a polynomial ring with coefficients in $R$ with respect to the set of indeterminates $\left\{x_{1}, \ldots, x_{n}\right\}$, then $A=\sigma(R)\left\langle x_{1}, \ldots, x_{n}\right\rangle$ is a skew $P B W$ extension if and only if the following conditions hold:

(1) for each $x^{\alpha} \in \operatorname{Mon}(A)$ and every $0 \neq r \in R$, there exist unique elements $r_{\alpha}:=\sigma^{\alpha}(r) \in R \backslash\{0\}, p_{\alpha, r} \in A$, such that $x^{\alpha} r=r_{\alpha} x^{\alpha}+$ $p_{\alpha, r}$, where $p_{\alpha, r}=0$, or $\operatorname{deg}\left(p_{\alpha, r}\right)<|\alpha|$ if $p_{\alpha, r} \neq 0$. If $r$ is left invertible, so is $r_{\alpha}$.

(2) For each $x^{\alpha}, x^{\beta} \in \operatorname{Mon}(A)$, there exist unique elements $c_{\alpha, \beta} \in R$ and $p_{\alpha, \beta} \in A$ such that $x^{\alpha} x^{\beta}=c_{\alpha, \beta} x^{\alpha+\beta}+p_{\alpha, \beta}$, where $d_{\alpha, \beta}$ is left invertible, $p_{\alpha, \beta}=0$, or $\operatorname{deg}\left(p_{\alpha, \beta}\right)<|\alpha+\beta|$ if $p_{\alpha, \beta} \neq 0$.

Remark 2 ([44], Proposition 2.9 and Remark 2.10). Consider $A=$ $\sigma(R)\left\langle x_{1}, \ldots, x_{n}\right\rangle$ a skew PBW extension over $R$.

(a) If $\alpha=\left(\alpha_{1}, \ldots, \alpha_{n}\right) \in \mathbb{N}^{n}$ and $r$ is an element of $R$, then

$$
\begin{aligned}
x^{\alpha} r= & x_{1}^{\alpha_{1}} x_{2}^{\alpha_{2}} \cdots x_{n-1}^{\alpha_{n-1}} x_{n}^{\alpha_{n}} r \\
= & x_{1}^{\alpha_{1}} \cdots x_{n-1}^{\alpha_{n-1}}\left(\sum_{j=1}^{\alpha_{n}} x_{n}^{\alpha_{n}-j} \delta_{n}\left(\sigma_{n}^{j-1}(r)\right) x_{n}^{j-1}\right) \\
& +x_{1}^{\alpha_{1}} \cdots x_{n-2}^{\alpha_{n-2}}\left(\sum_{j=1}^{\alpha_{n-1}} x_{n-1}^{\alpha_{n-1}-j} \delta_{n-1}\left(\sigma_{n-1}^{j-1}\left(\sigma_{n}^{\alpha_{n}}(r)\right)\right) x_{n-1}^{j-1}\right) x_{n}^{\alpha_{n}} \\
& +x_{1}^{\alpha_{1}} \cdots x_{n-3}^{\alpha_{n-3}}\left(\sum_{j=1}^{\alpha_{n-2}} x_{n-2}^{\alpha_{n-2}-j} \delta_{n-2}\left(\sigma_{n-2}^{j-1}\left(\sigma_{n-1}^{\alpha_{n-1}}\left(\sigma_{n}^{\alpha_{n}}(r)\right)\right)\right) x_{n-2}^{j-1}\right) \\
& \quad \times x_{n-1}^{\alpha_{n-1}} x_{n}^{\alpha_{n}} \\
& +\cdots+x_{1}^{\alpha_{1}}\left(\sum_{j=1}^{\alpha_{2}} x_{2}^{\alpha_{2}-j} \delta_{2}\left(\sigma_{2}^{j-1}\left(\sigma_{3}^{\alpha_{3}}\left(\sigma_{4}^{\alpha_{4}}\left(\cdots\left(\sigma_{n}^{\alpha_{n}}(r)\right)\right)\right)\right)\right) x_{2}^{j-1}\right) \\
& \quad \times x_{3}^{\alpha_{3}} x_{4}^{\alpha_{4}} \cdots x_{n-1}^{\alpha_{n-1}} x_{n}^{\alpha_{n}} \\
& +\sigma_{1}^{\alpha_{1}}\left(\sigma_{2}^{\alpha_{2}}\left(\cdots\left(\sigma_{n}^{\alpha_{n}}(r)\right)\right)\right) x_{1}^{\alpha_{1}} \cdots x_{n}^{\alpha_{n}}, \\
\sigma_{j}^{0}:= & \operatorname{id}_{R} \quad \text { for } 1 \leqslant j \leqslant n .
\end{aligned}
$$

(b) If $a_{i}, b_{j} \in R$ and $X_{i}:=x_{1}^{\alpha_{i 1}} \cdots x_{n}^{\alpha_{i n}}, Y_{j}:=x_{1}^{\beta_{j 1}} \cdots x_{n}^{\beta_{j n}}$, when we compute every summand of $a_{i} X_{i} b_{j} Y_{j}$ we obtain products of the coefficient $a_{i}$ with several evaluations of $b_{j}$ in $\sigma$ 's and $\delta$ 's depending on the coordinates of $\alpha_{i}$. 


\section{2. $\quad \Sigma$-rigid rings and $(\Sigma, \Delta)$-compatible rings}

In this section, we consider some results concerning $\Sigma$-rigid rings and $(\Sigma, \Delta)$-compatible rings, and their relation with skew PBW extensions. For the next definition, consider the notation in Remark 1 (i).

Definition 2 ([44], Definition 3.2). Let $R$ be a ring and $\Sigma$ a non-empty and finite family of endomorphisms of $R . \Sigma$ is called a rigid endomorphisms family if $r \sigma^{\alpha}(r)=0$ implies $r=0$, where $r \in R$ and $\alpha \in \mathbb{N}^{n} . R$ is said to be $\Sigma$-rigid if there exists a rigid endomorphisms family $\Sigma$ of $R$.

The motivation to define $\Sigma$-rigid rings was to generalize the rigid rings introduced by Krempa [25]. Now, if $\Sigma$ is a rigid endomorphisms family of $R$, then every element $\sigma_{i} \in \Sigma$ is a monomorphism. In fact, $\Sigma$-rigid rings are reduced rings: if $R$ is a $\Sigma$-rigid ring and $r^{2}=0$ for $r \in R$, then we have the equalities $0=r \sigma^{\alpha}\left(r^{2}\right) \sigma^{\alpha}\left(\sigma^{\alpha}(r)\right)=r \sigma^{\alpha}(r) \sigma^{\alpha}(r) \sigma^{\alpha}\left(\sigma^{\alpha}(r)\right)=$ $r \sigma^{\alpha}(r) \sigma^{\alpha}\left(r \sigma^{\alpha}(r)\right)$, i.e., $r \sigma^{\alpha}(r)=0$ and so $r=0$, that is, $R$ is reduced. With this in mind, we consider the finite family of injective endomorphisms $\Sigma$ and the family $\Delta$ of $\Sigma$-derivations in a skew PBW extension $A$ over $R$ (Proposition 1). The notion of rigidness with another ring theoretical properties such as minimal prime ideals, Armendariz, McCoy, Baer, quasiBaer, p. p and p. q have been investigated for skew PBW extensions (c.f. Reyes et al. [40], [45], [50]).

Recall that if $A$ is a skew PBW extension of $R$ where the the elements $d_{i, j}$ are invertible in $R$, then $R$ is $\Sigma$-rigid if and only if $A$ is a reduced ring, see [44], Proposition 3.5.

Proposition 3 ([44], Lemma 3.3 and Corollary 3.4). If $R$ is a $\Sigma$-rigid ring and $a, b \in R$, then:

(1) If $a b=0$ then $a \sigma^{\alpha}(b)=\sigma^{\alpha}(a) b=0$, for any $\alpha \in \mathbb{N}^{n}$.

(2) If $a b=0$ then $a \delta^{\beta}(b)=\delta^{\beta}(a) b=0$, for any $\beta \in \mathbb{N}^{n}$.

(3) If $a b=0$ then $a \sigma^{\alpha}\left(\delta^{\beta}(b)\right)=a \delta^{\beta}\left(\sigma^{\alpha}(b)\right)=0$, for every $\alpha, \beta \in \mathbb{N}^{n}$.

(4) If $a \sigma^{\theta}(b)=\sigma^{\theta}(a) b=0$ for some $\theta \in \mathbb{N}^{n}$, then $a b=0$.

(5) If $A$ is a skew $P B W$ extension over $R$, the equality ab $=0$ implies $a x^{\alpha} b x^{\beta}=0$, for any elements $a, b \in R$ and every $\alpha, \beta \in \mathbb{N}^{n}$.

Next, we present the notion of $(\Sigma, \Delta)$-compatible rings which was introduced independently by Hashemi et al. [17] and the authors [48].

Definition 3 ([17], Definition 3.1; [48], Definition 3.2). Consider a ring $R$ with a finite family of endomorphisms $\Sigma$ and a finite family of $\Sigma$-derivations $\Delta$. Following the notation established in Remark 1 (i), we have: $R$ is said to 
be $\Sigma$-compatible if for each $a, b \in R, a \sigma^{\alpha}(b)=0$ if and only if $a b=0$, where $\alpha \in \mathbb{N}^{n} ; R$ is said to be $\Delta$-compatible if for each $a, b \in R, a b=0$ implies $a \delta^{\beta}(b)=0$, where $\beta \in \mathbb{N}^{n}$. If $R$ is both $\Sigma$-compatible and $\Delta$-compatible, then $R$ is called $(\Sigma, \Delta)$-compatible.

Remark 3. - From [17], Lemma 3.5 or [48], Proposition 3.4, we know that every $\Sigma$-rigid ring is a $(\Sigma, \Delta)$-compatible ring. The converse is false as we can see in [48], Example 3.6. Nevertheless, both notions coincide when the ring is assumed to be reduced: if $A=\sigma(R)\left\langle x_{1}, \ldots, x_{n}\right\rangle$ is a skew PBW extension over $R$, then the following statements are equivalent: (1) $R$ is reduced and $(\Sigma, \Delta)$ compatible. (2) $R$ is $\Sigma$-rigid. (3) $A$ is reduced ([17], Lemma 3.5; [48], Theorem 3.9).

- $(\Sigma, \Delta)$-compatible rings extend the compatible rings defined by Annin [3] and the notion of $(\sigma, \delta)$-compatible ring introduced in [16]. As a matter of fact, $(\Sigma, \Delta)$-compatible rings have been very useful in the characterization of different radicals (Wedderburn radical, lower nil radical, Levitzky radical, upper nil radical, the set of all nilpotent elements, the sum of all nil left ideals) and another ring and module theoretical properties of skew PBW extensions (e.g., Hashemi et al. [15], [18], [19], and Reyes and Suárez [45], [50]).

Proposition 4 ([17], Lemma 3.3; [48], Proposition 3.8). Let $R$ be a $(\Sigma, \Delta)$-compatible ring. For every $a, b \in R$, we have:

(1) if $a b=0$, then $a \sigma^{\theta}(b)=\sigma^{\theta}(a) b=0$, for each $\theta \in \mathbb{N}^{n}$.

(2) If $\sigma^{\beta}(a) b=0$ for some $\beta \in \mathbb{N}^{n}$, then $a b=0$.

(3) If $a b=0$, then $\sigma^{\theta}(a) \delta^{\beta}(b)=\delta^{\beta}(a) \sigma^{\theta}(b)=0$, for every $\theta, \beta \in \mathbb{N}^{n}$.

Different examples of skew PBW extensions over $(\Sigma, \Delta)$-compatible rings can be found in [17], [45], and [49].

\section{Weak symmetric rings}

Ouyang and Chen [42], Definition 1, introduced the notion of weak symmetric ring in the following way: a ring $R$ is called a weak symmetric ring if $a b c \in \operatorname{nil}(R)$ implies $a c b \in \operatorname{nil}(R)$, for every elements $a, b, c \in R$. They proved that this notion extends the concept of symmetric ring, that is, all symmetric rings are weak symmetric ([42], Proposition 2.1). However, the converse of the assertion is false, i.e., there exists a weak symmetric ring which is not symmetric ([42], Example 2.2).

With the aim of studying these notions of symmetry in the case of skew PBW extensions, we start with four results (Lemmas 1 and 2 and 
Theorems 1 and 2) about nilpotent elements in skew PBW extensions. Our Lemmas 1 and 2 generalize [42], Lemmas 2.7 and 2.8, respectively.

Lemma 1. If $R$ is a $(\Sigma, \Delta)$-compatible and reversible ring and $a, b \in R$, then $a b \in \operatorname{nil}(R)$ implies that $a \sigma^{\alpha}\left(\delta^{\beta}(b)\right)$ and $a \delta^{\beta}\left(\sigma^{\alpha}(b)\right)$ are elements of $\operatorname{nil}(R)$, where $\alpha, \beta \in \mathbb{N}^{n}$.

Proof. We follow the arguments presented in [42], Lemma 2.7. By assumption, there exists a positive integer $k$ such that $(a b)^{k}=0$. Consider the following equalities:

$$
\begin{aligned}
(a b)^{k} & =a b a b \cdots a b a b a b \quad(k \text { times }) \\
& =a b a b \cdots a b a b a \sigma^{\alpha}\left(\delta^{\beta}(b)\right) \quad(\text { Proposition 4 }(3)) \\
& =a \sigma^{\alpha}\left(\delta^{\beta}(b)\right) a b a b a b \cdots a b a b \quad(R \text { is reversible }) \\
& =a \sigma^{\alpha}\left(\delta^{\beta}(b)\right) a b a b \cdots a b a \sigma^{\alpha}\left(\delta^{\beta}(b)\right) \quad(\text { Proposition } 4(3)) \\
& =a \sigma^{\alpha}\left(\delta^{\beta}(b)\right) a \sigma^{\alpha}\left(\delta^{\beta}(b)\right) a b a b \cdots a b \quad(R \text { is reversible })
\end{aligned}
$$

Continuing in this way, we guarantee that the element $a \sigma^{\alpha}\left(\delta^{\beta}(b)\right)$ belongs to $\operatorname{nil}(R)$. For the element $a \delta^{\beta}\left(\sigma^{\alpha}(b)\right)$ the reasoning is completely similar.

Lemma 2. If $R$ is a $(\Sigma, \Delta)$-compatible ring and $a, b \in R$, then $a \sigma^{\theta}(b) \in$ $\operatorname{nil}(R)$ implies $a b \in \operatorname{nil}(R)$, where $\theta \in \mathbb{N}^{n}$.

Proof. Following the argument developed in [42], Lemma 2.8, since we have that $a \sigma^{\theta}(b) \in \operatorname{nil}(R)$, then there exists a positive integer $k$ satisfying $\left(a \sigma^{\theta}(b)\right)^{k}=0$. We have the following assertions:

$$
\begin{aligned}
\left(a \sigma^{\theta}(b)\right)^{k} & =a \sigma^{\theta}(b) a \sigma^{\theta}(b) \cdots a \sigma^{\theta}(b) a \sigma^{\theta}(b) \quad(k \text { times }) \\
& =a \sigma^{\theta}(b) a \sigma^{\theta}(b) \cdots a \sigma^{\theta}(b) a b \quad(\Sigma \text {-compatibility) } \\
& =a \sigma^{\theta}(b) a \sigma^{\theta}(b) \cdots a \sigma^{\theta}(b) \sigma^{\theta}(a b) \quad \text { (Proposition 4 (1)) } \\
& =a \sigma^{\theta}(b) a \sigma^{\theta}(b) \cdots a \sigma^{\theta}(b a b) \quad\left(\sigma^{\theta} \text { is an endomorphism of } R\right) \\
& =a \sigma^{\theta}(b) a \sigma^{\theta}(b) \cdots a b a b \quad \text { (Definition of } \Sigma \text {-compatibility). }
\end{aligned}
$$

If we continue in this way, we can see that the element $a b \in \operatorname{nil}(R)$, which concludes the proof.

We recall from Liu and Zhao [34], Lemma 3.1, that if $R$ is a semicommutative ring, then $\operatorname{nil}(R)$ is an ideal of $R$. Our Theorem 1 generalizes [42], Lemma 2.10. We need to assume that the elements $d_{i, j}$ of Definition 1 (iv) 
are central in $R$. With the purpose of abbreviating, we will write o.t.l.t to mean other terms less than in the sense of monomial orders (Definition 1 (ii)).

Theorem 1. If $A=\sigma(R)\left\langle x_{1}, \ldots, x_{n}\right\rangle$ is a skew PBW extension over $a(\Sigma, \Delta)$-compatible and reversible ring $R$, then for every element $f=$ $\sum_{i=0}^{m} a_{i} X_{i} \in A, f \in \operatorname{nil}(A)$ if and only if $a_{i} \in \operatorname{nil}(R)$, for each $0 \leqslant i \leqslant m$.

Proof. We fix a total order $\preceq$ on $\operatorname{Mon}(A)$. Let $f \in A$ be given as above and suppose that $f \in \operatorname{nil}(A)$ with $X_{1} \prec X_{2} \prec \cdots \prec X_{m}$. Consider the notation established in Proposition 2. There exists a positive integer $k$ such that $f^{k}=\left(a_{0}+a_{1} X_{1}+\cdots+a_{m} X_{m}\right)^{k}=0$. Inductively, one can see that for $f^{k}$,

$$
f^{k}=\left\{a_{m} \prod_{l=1}^{k-1} \sigma^{l \alpha_{m}}\left(a_{m}\right) d_{l \alpha_{m}, \alpha_{m}} x^{k \alpha_{m}}\right\}+\text { o.t.l.t } \exp \left(x^{k \alpha_{m}}\right),
$$

whence $0=\operatorname{lc}\left(f^{k}\right)=a_{m} \prod_{l=1}^{k-1} \sigma^{l \alpha_{m}}\left(a_{m}\right) d_{l \alpha_{m}, \alpha_{m}}$, and since the elements $d$ 's are central in $R$ and left invertible, $0=\operatorname{lc}\left(f^{k}\right)=a_{m} \prod_{l=1}^{k-1} \sigma^{l \alpha_{m}}\left(a_{m}\right)$. Using the $\Sigma$-compatibility of $R$, we obtain $a_{m} \in \operatorname{nil}(R)$. Now, since

$$
\begin{aligned}
f^{k}= & \left(\left(a_{0}+a_{1} X_{1}+\cdots+a_{m-1} X_{m-1}\right)+a_{m} X_{m}\right)^{k} \\
= & \left(\left(a_{0}+a_{1} X_{1}+\cdots+a_{m-1} X_{m-1}\right)+a_{m} X_{m}\right) \\
& \quad \times\left(\left(a_{0}+a_{1} X_{1}+\cdots+a_{m-1} X_{m-1}\right)+a_{m} X_{m}\right) \\
& \quad \cdots\left(\left(a_{0}+a_{1} X_{1}+\cdots+a_{m-1} X_{m-1}\right)+a_{m} X_{m}\right) \quad(k \text { times }) \\
= & \left(a_{0}+a_{1} X_{1}+\cdots+a_{m-1} X_{m-1}\right)^{2} \\
& \quad+\left(a_{0}+a_{1} X_{1}+\cdots+a_{m-1} X_{m-1}\right) a_{m} X_{m} \\
& \left.+a_{m} X_{m}\left(a_{0}+a_{1} X_{1}+\cdots+a_{m-1} X_{m-1}\right)+a_{m} X_{m} a_{m} X_{m}\right] \\
& \quad \cdots\left(\left(a_{0}+a_{1} X_{1}+\cdots+a_{m-1} X_{m-1}\right)+a_{m} X_{m}\right) \\
= & \left(a_{0}+a_{1} X_{1}+\cdots+a_{m-1} X_{m-1}\right)^{k}+h,
\end{aligned}
$$

where $h$ is an element of $A$ which involves products of monomials with the term $a_{m} X_{m}$ on the left and the right, by Remark 2 and having in mind that $a_{m} \in \operatorname{nil}(R)$, which is an ideal of $R$ (remember that reversible implies semicommutative), the expression for $f^{k}$ reduces to $f^{k}=\left(a_{0}+\right.$ $\left.a_{1} X_{1}+\cdots+a_{m-1} X_{m-1}\right)^{k}$. Using a similar reasoning as above, one can prove that

$$
f^{k}=a_{m-1} \prod_{l=1}^{k-1} \sigma^{l\left(\alpha_{m-1}\right)}\left(a_{m-1}\right) d_{l\left(\alpha_{m-1}\right), \alpha_{m-1}} x^{k \alpha_{m-1}}+\text { o.t.l.t } \exp \left(x^{k \alpha_{m-1}}\right) .
$$


Hence $\operatorname{lc}\left(f^{k}\right)=a_{m-1} \prod_{l=1}^{k-1} \sigma^{l \alpha_{m-1}}\left(a_{m-1}\right) d_{l \alpha_{m-1}, \alpha_{m-1}}$, and so $a_{m-1} \in$ $\operatorname{nil}(R)$. If we repeat this argument, it follows that $a_{i} \in \operatorname{nil}(R)$, for $0 \leqslant i \leqslant$ $m$.

Conversely, suppose that $a_{i} \in \operatorname{nil}(R)$, for every $i$. If $k_{i}$ is the minimum integer positive such that $a_{i}^{k_{i}}=0$, for every $i$, let $k:=\max \left\{k_{i} \mid 1 \leqslant i \leqslant n\right\}$. It is clear that $a_{i}^{k}=0$, for all $i$. Let us prove that $f^{(m+1) k+1}=0$, and hence, $f \in \operatorname{nil}(A)$. Since the expression for $f$ have $m+1$ terms, when we realize the product $f^{(m+1) k+1}$ we have sums of products of the form

$$
a_{i, 1} X_{i, 1} a_{i, 2} X_{i, 2} \cdots a_{i,(m+1) k} X_{i,(m+1) k} a_{i,(m+1) k+1} X_{i,(m+1) k+1} .
$$

Note that there are exactly $(m+1)^{(m+1) k+1}$ products of the form $(1)$. Now, since when we compute $f^{(m+1) k+1}$ every product as (1) involves at least $k$ elements $a_{i}$, for some $i$, then every one of these products is equal to zero by Remark 2 and the $(\Sigma, \Delta)$-compatibility of $R$ (more exactly, Proposition 4). In this way, every term of $f^{(m+1) k+1}$ is equal to zero, and hence $f \in \operatorname{nil}(A)$.

The next theorem generalizes [42], Theorem 2.11. Let nil $(R) A:=\{f \in$ $\left.A \mid f=a_{0}+a_{1} X_{1}+\cdots+a_{m} X_{m}, a_{i} \in \operatorname{nil}(R)\right\}$.

Theorem 2. Let $A=\sigma(R)\left\langle x_{1}, \ldots, x_{n}\right\rangle$ be a skew $P B W$ extension over a reversible and $(\Sigma, \Delta)$-compatible ring $R$. If we have the elements $f=$ $\sum_{i=0}^{m} a_{i} X_{i}, g=\sum_{j=0}^{t} b_{j} Y_{j}$, and $h=\sum_{k=0}^{l} c_{k} Z_{k}$ of $A$, and $r$ is any element of $R$, then we have the following assertions:

(1) $f g \in \operatorname{nil}(A) \Leftrightarrow a_{i} b_{j} \in \operatorname{nil}(R)$, for all $i, j$.

(2) $f g r \in \operatorname{nil}(A) \Leftrightarrow a_{i} b_{j} r \in \operatorname{nil}(R)$, for all $i, j$.

(3) $f g h \in \operatorname{nil}(A) \Leftrightarrow a_{i} b_{j} c_{k} \in \operatorname{nil}(R)$, for all $i, j, k$.

Proof. Again, we fix a total order on $\operatorname{Mon}(A)$. (1) As we see in the proof of Theorem 1, $\operatorname{nil}(A) \subseteq \operatorname{nil}(R) A$. With this in mind, consider two elements $f, g \in A$ given by $f=\sum_{i=0}^{m} a_{i} X_{i}$ and $g=\sum_{j=0}^{t} b_{j} Y_{j}$ with $f g \in \operatorname{nil}(A)$. Let $X_{i}:=x_{1}^{\alpha_{i 1}} \cdots x_{n}^{\alpha_{i n}}, Y_{j}:=x_{1}^{\beta_{j 1}} \cdots x_{n}^{\beta_{j n}}$, for all $i, j$. We have

$$
f g=\sum_{k=0}^{m+t}\left(\sum_{i+j=k} a_{i} X_{i} b_{j} Y_{j}\right) \in \operatorname{nil}(A) \subseteq \operatorname{nil}(R) A,
$$

and $\operatorname{lc}(f g)=a_{m} \sigma^{\alpha_{m}}\left(b_{t}\right) d_{\alpha_{m}, \beta_{t}} \in \operatorname{nil}(R)$. Since the elements $d_{i, j}$ are in the center of $R$, then $d_{\alpha_{m}, \beta_{t}}$ are also in the center of $R$, whence $a_{m} \sigma^{\alpha_{m}}\left(b_{t}\right) \in$ $\operatorname{nil}(R)$, and by Lemma 2 it follows that $a_{m} b_{t} \in \operatorname{nil}(R)$. The idea is to prove that $a_{p} b_{q} \in \operatorname{nil}(R)$, for $p+q \geqslant 0$. We proceed by induction. Suppose 
that $a_{p} b_{q} \in \operatorname{nil}(R)$, for $p+q=m+t, m+t-1, m+t-2, \ldots, k+1$, for some $k>0$. By Lemma 1 , we obtain $a_{p} X_{p} b_{q} Y_{q} \in \operatorname{nil}(R) A$ for these values of $p+q$. In this way, it is sufficient to consider the sum of the products $a_{u} X_{u} b_{v} Y_{v}$, where $u+v=k, k-1, k-2, \ldots, 0$. Fix $u$ and $v$. Consider the sum of all terms of $f g$ having exponent $\alpha_{u}+\beta_{v}$. From Remark 2 and the assumption $f g \in \operatorname{nil}(A)$, we know that the sum of all coefficients of all these terms can be written as

$$
\begin{aligned}
& a_{u} \sigma^{\alpha_{u}}\left(b_{v}\right) d_{\alpha_{u}, \beta_{v}} \\
& +\sum_{\alpha_{u^{\prime}}+\beta_{v^{\prime}}=\alpha_{u}+\beta_{v}} a_{u^{\prime}} \sigma^{\alpha_{u^{\prime}}}\left(\sigma^{\prime} \text { s and } \delta^{\prime} \text { s evaluated in } b_{v^{\prime}}\right) d_{\alpha_{u^{\prime}}, \beta_{v^{\prime}}} \in \operatorname{nil}(R) \text {. }
\end{aligned}
$$

As we assumed before, $a_{p} b_{q} \in \operatorname{nil}(R)$ for $p+q=m+t, m+t-1, \ldots, k+1$, so Lemma 1 guarantees that the product $a_{p}\left(\sigma^{\prime}\right.$ s and $\delta^{\prime}$ s evaluated in $\left.b_{q}\right)$, for any order of $\sigma^{\prime} \mathrm{s}$ and $\delta^{\prime} \mathrm{s}$, is an element of nil $(R)$. Since $R$ is reversible, then $\left(\sigma^{\prime}\right.$ s and $\delta^{\prime}$ s evaluated in $\left.b_{q}\right) a_{p} \in \operatorname{nil}(R)$. In this way, multiplying (2) on the right by $a_{k}$, and using the fact that the elements $d$ 's are in the center of $R$, we obtain that the sum

$$
\begin{aligned}
& a_{u} \sigma^{\alpha_{u}}\left(b_{v}\right) a_{k} d_{\alpha_{u}, \beta_{v}} \\
& \quad+\sum_{\alpha_{u^{\prime}}+\beta_{v^{\prime}}=\alpha_{u}+\beta_{v}} a_{u^{\prime}} \sigma^{\alpha_{u^{\prime}}}\left(\sigma^{\prime} \text { s and } \delta^{\prime} \text { s evaluated in } b_{v^{\prime}}\right) a_{k} d_{\alpha_{u^{\prime}}, \beta_{v^{\prime}}}
\end{aligned}
$$

is an element of $\operatorname{nil}(R)$, whence, $a_{u} \sigma^{\alpha_{u}}\left(b_{0}\right) a_{k} \in \operatorname{nil}(R)$. Since $u+v=k$ and $v=0$, then $u=k$, so $a_{k} \sigma^{\alpha_{k}}\left(b_{0}\right) a_{k} \in \operatorname{nil}(R)$, from which $a_{k} \sigma^{\alpha_{k}}\left(b_{0}\right) \in \operatorname{nil}(R)$ and hence $a_{k} b_{0} \in \operatorname{nil}(R)$ by Lemma 2 . Therefore, we now have to study the expression (2) for $0 \leqslant u \leqslant k-1$ and $u+v=k$. If we multiply (3) on the right by $a_{k-1}$, then

$$
\begin{aligned}
& a_{u} \sigma^{\alpha_{u}}\left(b_{v}\right) a_{k-1} d_{\alpha_{u}, \beta_{v}} \\
& \quad+\sum_{\alpha_{u^{\prime}}+\beta_{v^{\prime}}=\alpha_{u}+\beta_{v}} a_{u^{\prime}} \sigma^{\alpha_{u^{\prime}}}\left(\sigma^{\prime} \text { s and } \delta^{\prime} \text { s evaluated in } b_{v^{\prime}}\right) a_{k-1} d_{\alpha_{u^{\prime}}, \beta_{v^{\prime}}}
\end{aligned}
$$

is also an element of $\operatorname{nil}(R)$. Using a similar reasoning as above, we can see that the element $a_{u} \sigma^{\alpha_{u}}\left(b_{1}\right) a_{k-1} d_{\alpha_{u}, \beta_{1}}$ belongs to nil $(R)$. Since the elements $d$ 's are central and left invertible, $a_{u} \sigma^{\alpha_{u}}\left(b_{1}\right) a_{k-1} \in \operatorname{nil}(R)$, and using the fact $u=k-1$, we have $a_{k-1} \sigma^{\alpha_{k-1}}\left(b_{1}\right) \in \operatorname{nil}(R)$, from which $a_{k-1} b_{1} \in \operatorname{nil}(R)$. Continuing in this way we prove that $a_{i} b_{j} \in \operatorname{nil}(R)$, for $i+j=k$. Therefore $a_{i} b_{j} \in \operatorname{nil}(R)$, for $0 \leqslant i \leqslant m$ and $0 \leqslant j \leqslant t$.

Conversely, for the elements $f, g$ above, suppose that $a_{i} b_{j} \in \operatorname{nil}(R)$. From Lemma 1 we know that $a \sigma^{\alpha}\left(\delta^{\beta}(b)\right)$ and $a \delta^{\beta}\left(\sigma^{\alpha}(b)\right)$ are elements 
of $\operatorname{nil}(R)$, for every $\alpha, \beta \in \mathbb{N}^{n}$. Now, Remark 2 implies that when we compute every summand of $a_{i} X_{i} b_{j} Y_{j}$ we obtain products of the coefficient $a_{i}$ with several evaluations of $b_{j}$ in $\sigma^{\prime}$ 's and $\delta$ 's depending of the coordinates of $\alpha_{i}$, and since $a \sigma_{i}\left(\delta^{\beta_{i}}(b)\right)$ and $a \delta^{\beta_{i}}\left(\sigma^{\alpha_{i}}(b)\right)$ are elements of $\operatorname{nil}(R)$, then every coefficient of each term of the expansion $f g$ given by $f g=\sum_{k=0}^{m+t}\left(\sum_{i+j=k} a_{i} X_{i} b_{j} Y_{j}\right)$ is an element of nil $(R)$. Therefore, Theorem 1 implies that the product $f g$ is an element of $R$.

(2) Let $g=b_{0}+b_{1} Y_{1}+\cdots+b_{t} Y_{t}$ be an element of $A$ with $Y_{t} \succ \cdots \succ Y_{1}$. Then

$$
\begin{aligned}
g r & =\left(b_{0}+b_{1} Y_{1}+\cdots+b_{t} Y_{t}\right) r=b_{0} r+b_{1} Y_{1} r+\cdots+b_{t} Y_{t} r \\
& =b_{0} r+b_{1}\left(\sigma^{\beta_{1}}(r) Y_{1}+p_{\beta_{1}, r}\right)+\cdots+b_{t}\left(\sigma^{\beta_{t}}(r) Y_{t}+p_{\beta_{t}, r}\right) \\
& =b_{0} r+b_{1} \sigma^{\beta_{1}}(r) Y_{1}+b_{1} p_{\beta_{1}, r}+\cdots+b_{t} \sigma^{\beta_{t}}(r) Y_{t}+b_{t} p_{\beta_{t}, r}
\end{aligned}
$$

where $p_{\beta_{j}, r}=0$, or $\operatorname{deg}\left(p_{\beta_{j}, r}\right)<|\alpha|$ if $p_{\beta_{j}, r} \neq 0$, for $j=1, \ldots, t$ (Proposition 2). Note that $\operatorname{lc}(g r)=b_{t} \sigma^{\beta_{t}}(r)$. Then,

$$
\begin{aligned}
f g r=( & \left.a_{0}+a_{1} X_{1}+\cdots+a_{m} X_{m}\right)\left(b_{0} r+b_{1} \sigma^{\beta_{1}}(r) Y_{1}+b_{1} p_{\beta_{1}, r}\right. \\
& \left.+\cdots+b_{t} \sigma^{\beta_{t}}(r) Y_{t}+b_{t} p_{\beta_{t}, r}\right) \\
=a_{0} & b_{0} r+a_{0} b_{1} \sigma^{\beta_{1}}(r) Y_{1}+a_{0} b_{1} p_{\beta_{1}, r}+\cdots+a_{0} b_{t} \sigma^{\beta_{t}}(r) Y_{t}+a_{0} b_{t} p_{\beta_{t}, r} \\
& +a_{1} X_{1} b_{0} r+a_{1} X_{1} b_{1} \sigma^{\beta_{1}}(r) Y_{1}+a_{1} X_{1} b_{1} p_{\beta_{1}, r} \\
& +\cdots+a_{1} X_{1} b_{t} \sigma^{\beta_{t}}(r) Y_{t}+a_{1} X_{1} b_{t} p_{\beta_{t}, r} \\
& +\cdots+a_{m} X_{m} b_{0} r+a_{m} X_{m} b_{1} \sigma^{\beta_{1}}(r) Y_{1}+a_{m} X_{m} b_{1} p_{\beta_{1}, r} \\
& +\cdots+a_{m} X_{m} b_{t} \sigma^{\beta_{t}}(r) Y_{t}+a_{m} X_{m} b_{t} p_{\beta_{t}, r}
\end{aligned}
$$

Equivalently,

$$
\begin{aligned}
& f g r=a_{0} b_{0} r+a_{0} b_{1} \sigma^{\beta_{1}}(r) Y_{1}+a_{0} b_{1} p_{\beta_{1}, r}+\cdots+a_{0} b_{t} \sigma^{\beta_{t}}(r) Y_{t}+a_{0} b_{t} p_{\beta_{t}, r} \\
& \quad+a_{1}\left[\sigma^{\alpha_{1}}\left(b_{0} r\right) X_{1}+p_{\alpha_{1}, b_{0} r}\right]+a_{1}\left[\sigma^{\alpha_{1}}\left(b_{1} \sigma^{\beta_{1}}(r)\right) X_{1}+p_{\alpha_{1}, b_{1} \sigma^{\beta_{1}(r)}}\right] Y_{1} \\
& \quad+a_{1}\left[\sigma^{\alpha_{1}}\left(b_{1}\right)+p_{\alpha_{1}, b_{1}}\right] p_{\beta_{1}, r}+\cdots+a_{1}\left[\sigma^{\alpha_{1}}\left(b_{t} \sigma^{\beta_{t}}(r)\right) X_{1}+p_{\alpha_{1}, b_{t} \sigma^{\beta_{t}}(r)}\right] Y_{t} \\
& \quad+a_{1}\left[\sigma^{\alpha_{1}}\left(b_{t}\right) X_{1}+p_{\alpha_{1}, b_{t}}\right] p_{\beta_{t}, r}+\cdots+a_{m}\left[\sigma^{\alpha_{m}}\left(b_{0} r\right)+p_{\alpha_{m}, b_{0} r}\right] \\
& \quad+a_{m}\left[\sigma^{\alpha_{m}}\left(b_{1} \sigma^{\beta_{1}}(r) X_{1}+p_{\alpha_{1}, b_{1} \sigma^{\beta_{1}}(r)}\right] Y_{1}+a_{m}\left[\sigma^{\alpha_{m}}\left(b_{1}\right) X_{m}+p_{\alpha_{m}, b_{1}}\right] p_{\beta_{1}, r}\right. \\
& \quad+\cdots+a_{m}\left[\sigma^{\alpha_{m}}\left(b_{t} \sigma^{\beta_{t}}(r)\right) X_{m}+p_{\alpha_{m}, b_{t} \sigma^{\beta_{t}}(r)}\right] Y_{t} \\
& \quad+a_{m}\left[\sigma^{\alpha_{m}}\left(b_{t}\right) X_{m}+p_{\alpha_{m}, b_{t}}\right] p_{\beta_{t}, r},
\end{aligned}
$$

whence $\operatorname{lc}(f g r)=a_{m} \sigma^{\alpha_{m}}\left(b_{t} \sigma^{\beta_{t}}(r)\right)$, and since $R$ is $\Sigma$-compatible, Lemma 2 implies that $a_{m} b_{t} r \in \operatorname{nil}(R)$. Now, Lemma 1 guarantees that every term 
of any polynomial containing the product $a_{m} b_{t} r$ in the expression above for $f g r$ is an element of $\operatorname{nil}(R) A$. In this way, using an monomial order we can repeat this argument for the next monomial of $f g r$ less than lc $(f g r)$, and continuing this process until the first monomial to obtain that the elements $a_{i} b_{j} r$ are in $\operatorname{nil}(R)$, for all $i, j$.

Conversely, suppose that $a_{i} b_{j} r \in \operatorname{nil}(R)$, for every $i, j$, as above. As we saw above,

$$
\begin{aligned}
g r & =\left(b_{0}+b_{1} Y_{1}+\cdots+b_{t} Y_{t}\right) r=b_{0} r+b_{1} Y_{1} r+\cdots+b_{t} Y_{t} r \\
& =b_{0} r+b_{1}\left(\sigma^{\beta_{1}}(r) Y_{1}+p_{\beta_{1}, r}\right)+\cdots+b_{t}\left(\sigma^{\beta_{t}}(r) Y_{t}+p_{\beta_{t}, r}\right) \\
& =b_{0} r+b_{1} \sigma^{\beta_{1}}(r) Y_{1}+b_{1} p_{\beta_{1}, r}+\cdots+b_{t} \sigma^{\beta_{t}}(r) Y_{t}+b_{t} p_{\beta_{t}, r}
\end{aligned}
$$

where $p_{\beta_{j}, r}=0$, or $\operatorname{deg}\left(p_{\beta_{j}, r}\right)<|\alpha|$ if $p_{\beta_{j}, r} \neq 0$, for $j=1, \ldots, t$. Since $a_{i} b_{j} r \in \operatorname{nil}(R)$, for every $i, j$, Lemma 1 implies that $a_{i} b_{j} \sigma^{\alpha}\left(\delta^{\beta}(r)\right)$ and $a_{i} b_{j} \delta^{\beta}\left(\sigma^{\alpha}(r)\right)$ are elements of nil $(R)$, for every $\alpha, \beta \in \mathbb{N}^{n}$. In this way, Remark 2 applied to expression above for the product $f g r$ implies that every one of these summands have coefficients in $\operatorname{nil}(R)$, and since $\operatorname{nil}(R)$ is an ideal of $R$ because $R$ is reversible, Theorem 1 shows that $f g r \in \operatorname{nil}(A)$.

(3) The equivalence follows from (1) and (2) considering the product $g h$ as the only element $p \in A$.

Remark 4. About Theorem 2 (1) we have the following observation. In [48], Definition 4.1, the authors introduced the following condition: if $A$ is a skew PBW extension of $R$, we say that $R$ satisfies the condition (SA1) if whenever $f g=0$ for $f=a_{0}+a_{1} X_{1}+\cdots+a_{m} X_{m}$ and $g=$ $b_{0}+b_{1} Y_{1}+\cdots+b_{t} Y_{t}$ elements of $A$, then $a_{i} b_{j}=0$, for every $i, j$. It is clear that Theorem 2 extends this condition.

The next theorem generalizes [42], Theorem 2.12.

Theorem 3. If $A=\sigma(R)\left\langle x_{1}, \ldots, x_{n}\right\rangle$ is a skew $P B W$ extension over a reversible and $(\Sigma, \Delta)$-compatible ring $R$, then $R$ is weak symmetric if and only if $A$ is weak symmetric.

Proof. Since a subring of a weak symmetric ring is also a weak symmetric ring, we will only prove one implication. Suppose that $R$ is a weak symmetric ring. If $f=\sum_{i=0}^{s} a_{i} X_{i}, g=\sum_{j=0}^{t} b_{j} Y_{j}$ and $h=\sum_{k=0}^{l} c_{k} Z_{k}$ are elements of $A$ with $f g h \in \operatorname{nil}(A)$, then Theorem 2 implies that $a_{i} b_{j} c_{k} \in \operatorname{nil}(r)$, for every $i, j, k$, and hence $a_{i} c_{k} b_{j} \in \operatorname{nil}(R)$, for each $i, j, k$, since $R$ is weak symmetric. Finally, Theorem 2 shows that $f h g \in \operatorname{nil}(A)$. 
Corollary 1. If $R$ is a $\Sigma$-rigid ring, then $R$ is weak symmetric if and only if $A$ is weak symmetric.

Proof. Since we have the implications reduced $\Rightarrow$ symmetric $\Rightarrow$ weak symmetric, then the assertion follows from Theorem 3 .

Corollary 2 ([42], Corollaries 2.13 and 2.14). Let $R$ be a reversible ring. Then we have the following:

(1) $R$ is weak symmetric if and only if $R[x]$ is weak symmetric.

(2) If $R$ is $\sigma$-compatible, then $R$ is weak symmetric if and only if $R[x ; \sigma]$ is weak symmetric.

(3) If $R$ is $\delta$-compatible, then $R$ is weak symmetric if and only if the differential polynomial ring $R[x ; \sigma]$ is weak symmetric.

(4) Let $\alpha$ be an endomorphism and $\delta$ and $\alpha$-derivation of $R$. If $R$ is $\alpha$-rigid, then $R$ is weak symmetric if and only if $R[x ; \alpha, \delta]$ is weak symmetric.

With the aim of establishing Theorems 4 and 6 , we need to formulate a criterion which allows us to extend the family $\Sigma$ of injective endomorphisms, and the family of $\Sigma$-derivations $\Delta$ of the ring $R$ to the ring $A$. For the next proposition consider the injective endomorphisms $\sigma_{i} \in \Sigma$, and the $\sigma_{i}$-derivations $\delta_{i} \in \Delta(1 \leqslant i \leqslant n)$ formulated in Proposition 1 (compare with Artamonov [4], where the derivations of skew PBW extensions were computed partially).

Proposition 5 ([47], Theorem 5.1). Let $A=\sigma(R)\left\langle x_{1}, \ldots, x_{n}\right\rangle$ be a skew PBW extension over $R$. Suppose that $\sigma_{i} \delta_{j}=\delta_{j} \sigma_{i}, \delta_{i} \delta_{j}=\delta_{j} \delta_{i}$, and $\delta_{k}\left(d_{i, j}\right)=\delta_{k}\left(r_{l}^{(i, j)}\right)=0$, for $1 \leqslant i, j, k, l \leqslant n$, where $d_{i, j}$ and $r_{l}^{(i, j)}$ are as in Definition 1. If $\overline{\sigma_{k}}: A \rightarrow A$ and $\overline{\delta_{k}}: A \rightarrow A$ are the functions given by $\overline{\sigma_{k}}(f):=\sigma_{k}\left(a_{0}\right)+\sigma_{k}\left(a_{1}\right) X_{1}+\cdots+\sigma_{k}\left(a_{m}\right) X_{m}$ and $\overline{\delta_{k}}(f):=\delta_{k}\left(a_{0}\right)+$ $\delta_{k}\left(a_{1}\right) X_{1}+\cdots+\delta_{k}\left(a_{m}\right) X_{m}$, for every $f=a_{0}+a_{1} X_{1}+\cdots+a_{m} X_{m} \in A$, respectively, and $\overline{\sigma_{k}}(r):=\sigma_{k}(r)$, for every $1 \leqslant k \leqslant n$ and each $r \in R$, then $\overline{\sigma_{k}}$ is an injective endomorphism of $A$ and $\overline{\delta_{k}}$ is a $\overline{\sigma_{k}}$-derivation of $A$, for each $k$. Let $\bar{\Sigma}:=\left\{\overline{\sigma_{1}}, \ldots, \overline{\sigma_{n}}\right\}$ and $\bar{\Delta}:=\left\{\overline{\delta_{1}}, \ldots, \overline{\delta_{n}}\right\}$.

With Proposition 5 in our hands, we formulate Theorem 4 which extends [42], Theorem 2.17. With this aim, consider the skew PBW extension $A^{\prime}$ induced by injective endomorphisms and derivations established in Proposition 5, i.e., $A^{\prime}=\sigma(A)\left\langle x_{1}^{\prime}, \ldots, x_{n}^{\prime}\right\rangle$. We remark that using algorithms established in Acosta et al. [1] or Fajardo et al. [11], one can prove that $A^{\prime}$ is a left free $A$-module considering adequate relations between the 
indeterminates $x_{1}^{\prime}, \ldots, x_{n}^{\prime}$, that is, $A^{\prime}$ is certainly a skew PBW extension over $A$. For the sets of injective endomorphisms $\bar{\Sigma}$ and $\bar{\Sigma}$-derivations $\bar{\Delta}$ formulated in Proposition 5, consider a definition of $(\bar{\Sigma}, \bar{\Delta})$-compatible in a similar way to the Definition 3. Suppose that the elements $d_{i, j}$ in Definition 1 (iv) are central in $R$, for all $i, j$.

Theorem 4. If $A=\sigma(R)\left\langle x_{1}, \ldots, x_{n}\right\rangle$ is a skew PBW extension over a $\Sigma$-rigid ring $R$, then $A$ is weak symmetric if and only if $A^{\prime}$ is weak symmetric.

Proof. As we saw in section 4 , if $R$ is $\Sigma$-rigid, then $R$ is reduced, or equivalently, $A$ is reduced whence $A$ is reversible. The aim is to show that $A$ is $(\bar{\Sigma}, \bar{\Delta})$-compatible. From [48], Theorem 3.9, we know that $R$ is $(\Sigma, \Delta)$-compatible.

We fix a total order on $\operatorname{Mon}(A)$. Consider elements $f=a_{0}+a_{1} X_{1}+$ $\cdots+a_{m} X_{m}, g=b_{0}+b_{1} Y_{1}+\cdots+b_{t} Y_{t}$ in $A$ with $f g=0$ and let us see that $a_{i} b_{j}=0$, for every $i, j$. Since

$$
\begin{aligned}
f g & =\left(a_{0}+a_{1} X_{1}+\cdots+a_{m} X_{m}\right)\left(b_{0}+b_{1} Y_{1}+\cdots+b_{t} Y_{t}\right) \\
& =\sum_{k=0}^{m+t}\left(\sum_{i+j=k} a_{i} X_{i} b_{j} Y_{j}\right),
\end{aligned}
$$

then $\operatorname{lc}(f g)=a_{m} \sigma^{\alpha_{m}}\left(b_{t}\right) d_{\alpha_{m}, \beta_{t}}=0$ whence $a_{m} \sigma^{\alpha_{m}}\left(b_{t}\right)=0\left(d_{\alpha_{m}, \beta_{b}}\right.$ are central and left invertible), and by Proposition $3(4), a_{m} b_{t}=0$. The idea is to prove that $a_{p} b_{q}=0$, for $p+q \geqslant 0$. We proceed by induction. Suppose that $a_{p} b_{q}=0$, for $p+q=m+t, m+t-1, m+t-2, \ldots, k+1$, for some $k>0$. By Proposition $3(5)$ we obtain $a_{p} X_{p} b_{q} Y_{q}=0$ for these values of $p+q$. In this way we only consider the sum of the products $a_{u} X_{u} b_{v} Y_{v}$, where $u+v=k, k-1, k-2, \ldots, 0$. Fix $u$ and $v$. Consider the sum of all terms of $f g$ having exponent $\alpha_{u}+\beta_{v}$. From Remark 2 and the assumption $f g=0$, the sum of all coefficients of all these terms can be written as

$$
\begin{aligned}
& a_{u} \sigma^{\alpha_{u}}\left(b_{v}\right) d_{\alpha_{u}, \beta_{v}} \\
& \quad+\sum_{\alpha_{u^{\prime}}+\beta_{v^{\prime}}=\alpha_{u}+\beta_{v}} a_{u^{\prime}} \sigma^{\alpha_{u^{\prime}}}\left(\sigma^{\prime} \text { s and } \delta^{\prime} \text { s evaluated in } b_{v^{\prime}}\right) d_{\alpha_{u^{\prime}}, \beta_{v^{\prime}}}=0 .
\end{aligned}
$$

By assumption, we know that $a_{p} b_{q}=0$ for $p+q=m+t, m+t-1, \ldots, k+1$. So, Proposition $3(3)$ guarantees that $a_{p}\left(\sigma^{\prime}\right.$ s and $\delta^{\prime}$ s evaluated in $\left.b_{q}\right)$, for any order of $\sigma^{\prime} s$ and $\delta$ 's, is equal to zero. In this way, the expression $\left[\left(\sigma^{\prime} \text { s and } \delta^{\prime} \text { s evaluated in } b_{q}\right) a_{p}\right]^{2}$ is equal to zero, and hence we obtain 
the equality $\left(\sigma^{\prime}\right.$ s and $\delta^{\prime}$ s evaluated in $\left.b_{q}\right) a_{p}=0$ ( $R$ is reduced). In this way, multiplying (4) by $a_{k}$, and using the fact that the elements $d_{i, j}$ in Definition 1 (iv) are in the center of $R$,

$$
\begin{aligned}
& a_{u} \sigma^{\alpha_{u}}\left(b_{v}\right) a_{k} d_{\alpha_{u}, \beta_{v}} \\
& \quad+\sum_{\alpha_{u^{\prime}}+\beta_{v^{\prime}}=\alpha_{u}+\beta_{v}} a_{u^{\prime}} \sigma^{\alpha_{u^{\prime}}}\left(\sigma^{\prime} \text { s and } \delta^{\prime} \text { s evaluated in } b_{v^{\prime}}\right) a_{k} d_{\alpha_{u^{\prime}}, \beta_{v^{\prime}}}=0
\end{aligned}
$$

whence, $a_{u} \sigma^{\alpha_{u}}\left(b_{0}\right) a_{k}=0$. Since $u+v=k$ and $v=0$, then $u=k$, so $a_{k} \sigma^{\alpha_{k}}\left(b_{0}\right) a_{k}=0$, i.e., $\left[a_{k} \sigma^{\alpha_{k}}\left(b_{0}\right)\right]^{2}=0$, from which $a_{k} \sigma^{\alpha_{k}}\left(b_{0}\right)=0$ and $a_{k} b_{0}=0$ by Proposition 3 (4). Therefore, we now have to study the expression (4) for $0 \leqslant u \leqslant k-1$ and $u+v=k$. If we multiply (5) by $a_{k-1}$ we obtain

$$
\begin{aligned}
& a_{u} \sigma^{\alpha_{u}}\left(b_{v}\right) a_{k-1} d_{\alpha_{u}, \beta_{v}} \\
& \quad+\sum_{\alpha_{u^{\prime}}+\beta_{v^{\prime}}=\alpha_{u}+\beta_{v}} a_{u^{\prime}} \sigma^{\alpha_{u^{\prime}}}\left(\sigma^{\prime} \text { s and } \delta^{\prime} \text { s evaluated in } b_{v^{\prime}}\right) a_{k-1} d_{\alpha_{u^{\prime}}, \beta_{v^{\prime}}}=0 .
\end{aligned}
$$

By using a similar reasoning, we can show that $a_{u} \sigma^{\alpha_{u}}\left(b_{1}\right) a_{k-1} d_{\alpha_{u}, \beta_{1}}=0$. Then $a_{u} \sigma^{\alpha_{u}}\left(b_{1}\right) a_{k-1}=0$, and using the fact $u=k-1,\left[a_{k-1} \sigma^{\alpha_{k-1}}\left(b_{1}\right)\right]^{2}=$ 0 , which imply $a_{k-1} \sigma^{\alpha_{k-1}}\left(b_{1}\right)=0$ ( $R$ is reduced), that is, $a_{k-1} b_{1}=0$. Continuing in this way we prove that $a_{i} b_{j}=0$ for $i+j=k$. Hence $a_{i} b_{j}=0$, for $0 \leqslant i \leqslant m$ and $0 \leqslant j \leqslant t$, and therefore $a_{i} \sigma^{\alpha}\left(b_{j}\right)=a_{i} \delta^{\beta}\left(b_{j}\right)=0$, for all $\alpha, \beta \in \mathbb{N}^{n}$, since $R$ is $(\Sigma, \Delta)$-compatible. In this way, when we consider the expressions

$$
\begin{aligned}
f \overline{\sigma^{\alpha}} & (g)=\left(a_{0}+a_{1} X_{1}+\cdots+a_{m} X_{m}\right)\left(\sigma^{\alpha}\left(b_{0}\right)+\sigma^{\alpha}\left(b_{1}\right) Y_{1}+\cdots+\sigma^{\alpha}\left(b_{t}\right) Y_{t}\right) \\
& =\sum_{k=0}^{m+t}\left(\sum_{i+j=k} a_{i} X_{i} \sigma^{\alpha}\left(b_{j}\right) Y_{j}\right) \\
& =\sum_{k=0}^{m+t}\left(\sum_{i+j=k} a_{i}\left[\sigma^{\alpha_{i}}\left(\sigma^{\alpha}\left(b_{j}\right)\right) X_{i}+p_{\alpha_{i}, \sigma^{\alpha}\left(b_{j}\right)}\right) Y_{j}\right) \\
& =\sum_{k=0}^{m+t}\left(\sum_{i+j=k} a_{i} \sigma^{\alpha_{i}}\left(\sigma^{\alpha}\left(b_{j}\right)\right) X_{i} Y_{j}+a_{i} p_{\alpha_{i}, \sigma^{\alpha}\left(b_{j}\right)} Y_{j}\right) \\
& =\sum_{k=0}^{m+t}\left(\sum_{i+j=k} a_{i} \sigma^{\alpha_{i}}\left(\sigma^{\alpha}\left(b_{j}\right)\right)\left[d_{\alpha_{i}, \beta_{j}} x^{\alpha_{i}+\beta_{j}}+p_{\alpha_{i}, \beta_{j}}\right]+a_{i} p_{\alpha_{i}, \sigma^{\alpha}\left(b_{j}\right)} Y_{j}\right)
\end{aligned}
$$


and

$$
\begin{aligned}
f \overline{\delta^{\beta}}(g) & =\left(a_{0}+a_{1} X_{1}+\cdots+a_{m} X_{m}\right)\left(\delta^{\beta}\left(b_{0}\right)+\delta^{\beta}\left(b_{1}\right) Y_{1}+\cdots+\delta^{\beta}\left(b_{t}\right) Y_{t}\right) \\
& =\sum_{k=0}^{m+t}\left(\sum_{i+j=k} a_{i} X_{i} \delta^{\beta}\left(b_{j}\right) Y_{j}\right) \\
& =\sum_{k=0}^{m+t}\left(\sum_{i+j=k} a_{i}\left[\sigma^{\alpha_{i}}\left(\delta^{\beta}\left(b_{j}\right)\right) X_{i}+p_{\alpha_{i}, \delta^{\beta}\left(b_{j}\right)}\right] Y_{j}\right),
\end{aligned}
$$

or equivalently,

$$
\begin{aligned}
f \overline{\delta^{\beta}}(g) & =\sum_{k=0}^{m+t}\left(\sum_{i+j=k} a_{i} \sigma^{\alpha_{i}}\left(\delta^{\beta}\left(b_{j}\right)\right) X_{i} Y_{j}+a_{i} p_{\alpha_{i}, \delta^{\beta}\left(b_{j}\right)} Y_{j}\right) \\
& =\sum_{k=0}^{m+t}\left(\sum_{i+j=k} a_{i} \sigma^{\alpha_{i}}\left(\delta^{\beta}\left(b_{j}\right)\right)\left[d_{\alpha_{i}, \beta_{j}} x^{\alpha_{i}+\beta_{j}}+p_{\alpha_{i}, \beta_{j}}\right]+a_{i} p_{\alpha_{i}, \delta^{\beta}\left(b_{j}\right)} Y_{j}\right),
\end{aligned}
$$

Remark 2 implies that $f \overline{\sigma^{\alpha}}(g)=f \overline{\delta^{\beta}}(g)=0$, for every $\alpha, \beta \in \mathbb{N}^{n}$. In a similar way, if we start with the equality $f \overline{\sigma^{\alpha}}(g)=0$, then we can show that $f g=0$, which means that $A$ is $(\Sigma, \Delta)$-compatible. In this way, since we have showed that $A$ is reversible and $(\bar{\Sigma}, \bar{\Delta})$-compatible, the assertion follows from Theorem 3.

\section{Weak $(\Sigma, \Delta)$-symmetric rings}

Ouyang and Chen [42], Definition 2, introduced the notion of weak $(\alpha, \delta)$-symmetric ring in the following way: a $\operatorname{ring} R$ with an endomorphism $\sigma$ and an $\sigma$-derivation $\delta$ is said to be weak $\sigma$-symmetric provided that $a b c \in \operatorname{nil}(R)$ if and only if $a c \sigma(b) \in \operatorname{nil}(R)$, for any elements $a, b, c \in R$. $R$ is said to be weak $\delta$-symmetric, if for $a, b, c \in R, a b c \in \operatorname{nil}(R)$ implies $a c \delta(b) \in \operatorname{nil}(R)$. If $R$ is both weak $\sigma$-symmetric and weak $\delta$-symmetric, $R$ is called a weak $(\Sigma, \Delta)$-symmetric ring. With respect to the relation between weak symmetric rings and weak $(\alpha, \delta)$-symmetric rings, there is an example of a weak symmetric ring which is not weak $(\alpha, \delta)$-symmetric, see [42], Example 3.2. Note that for every subring $S$ of a weak $(\alpha, \delta)$-symmetric ring $R$ which satisfies $\alpha(S) \subseteq S$ and $\delta(S) \subseteq S$, it follows that $S$ is also a weak $(\alpha, \delta)$-symmetric ring. With these definitions in mind, we present in a natural way the notion of weak $(\Sigma, \Delta)$-symmetric ring for a ring $R$ with a family of endomorphisms $\Sigma$ and a family of $\Sigma$-derivations $\Delta$.

Definition 4. Let $R$ be a ring with a finite family of endomorphisms of $R$ and a finite family of $\Sigma=\left\{\sigma_{1}, \ldots, \sigma_{n}\right\}$-derivations $\Delta=\left\{\delta_{1}, \ldots, \delta_{n}\right\} . R$ is 
called weak $\Sigma$-symmetric, if $a b c \in \operatorname{nil}(R)$ implies that $a c \sigma_{i}(b) \in \operatorname{nil}(R)$, for every $i$ and each elements $a, b, c \in R$. $R$ is said to be weak $\Delta$-symmetric, if $a b c \in \operatorname{nil}(R)$ implies $a c \delta_{i}(b) \in \operatorname{nil}(R)$, for every $i$ and each elements $a, b, c \in R$. In the case $R$ is both weak $\Sigma$-symmetric and weak $\Delta$-symmetric, we say that $R$ is a weak $(\Sigma, \Delta)$-symmetric ring.

Definition 5. If $R$ is a ring with a family of endomorphisms of $R$ and a family of $\Sigma=\left\{\sigma_{1}, \ldots, \sigma_{n}\right\}$-derivations $\Delta=\left\{\delta_{1}, \ldots, \delta_{n}\right\}$, then an ideal $I$ of $R$ is said to be an weak-symmetric ideal, if abc $\in \operatorname{nil}(R)$ implies that $a c \sigma_{i}(b), a c \delta_{i}(b) \in \operatorname{nil}(R)$, for each $i$ and every elements $a, b, c \in I$.

The next proposition extends [42], Proposition 3.6.

Proposition 6. If $R$ is an Abelian ring (i.e., every idempotent element is central) with $\sigma_{i}(e)=e$ and $\delta_{i}(e)=0$, for any idempotent element $e$ of $R$, where $\sigma_{i} \in \Sigma$ and $\delta_{i} \in \Delta$, then the following statements are equivalent:

(1) $R$ is a weak $(\Sigma, \Delta)$-symmetric ring.

(2) $e R$ and $(1-e) R$ are weak $(\Sigma, \Delta)$-symmetric ideals.

Proof. We use similar arguments to those presented in [42], Proposition 3.6. (1) $\Rightarrow(2)$ It is clear. (2) $\Rightarrow$ (1) Consider elements $a, b, c \in R$ with $a b c \in \operatorname{nil}(R)$. It follows that eaebec, $(1-e) a(1-e) b(1-e) c \in \operatorname{nil}(R)$. By assumption, $e R$ and $(1-e) R$ are weak $(\Sigma, \Delta)$-symmetric ideals, so $e a e c \sigma_{i}(e b)=e a c \sigma_{i}(b) \in \operatorname{nil}(R)$ and $(1-e) a(1-e) c \sigma_{i}((1-e) b)=(1-$ $e) a c \sigma_{i}(b) \in \operatorname{nil}(R)$. This fact shows that $a c \sigma_{i}(b) \in \operatorname{nil}(R)$, for every $i$, and hence $R$ is weak $\Sigma$-symmetric. Now, since for any $r \in R, \delta_{i}(e r)=$ $\sigma_{i}(e) \delta_{i}(r)+\delta_{i}(e) r=e \delta_{i}(r)$, for every $i$, the assumptions on $R$ imply that if $a b c \in \operatorname{nil}(R)$, then $e a(e b)(e c),(1-e) a(1-e) b(1-e) c \in \operatorname{nil}(R)$. Therefore $e a e c \delta_{i}(e b)=e a c \delta_{i}(b),(1-e) a(1-e) c \delta_{i}((1-e) b)=(1-e) a c \delta_{i}(b) \in \operatorname{nil}(R)$. In this way, $a c \delta_{i}(b) \in \operatorname{nil}(R)$, for every $i$, which means that $R$ is weak $\Delta$-symmetric, and so $R$ is weak $(\Sigma, \Delta)$-symmetric.

For the next theorem, Theorem 5, we need some preliminary facts and the Proposition 7 which concerns about quotients of skew PBW extensions: consider $A=\sigma(R)\left\langle x_{1}, \ldots, x_{n}\right\rangle$ a skew PBW extension over $R$. Let $\Sigma:=\left\{\sigma_{1}, \ldots, \sigma_{n}\right\}$ and $\Delta:=\left\{\delta_{1}, \ldots, \delta_{n}\right\}$ such as in Proposition 1 . Following [28], section 2, if $I$ is an ideal of $R, I$ is called $\Sigma$-invariant $(\Delta$-invariant $)$, if it is invariant under each injective endomorphism $\sigma_{i}$ $\left(\sigma_{i}\right.$-derivation $\left.\delta_{i}\right)$ of $\Sigma(\Delta)$, that is, $\sigma_{i}(I) \subseteq I\left(\delta_{i}(I) \subseteq I\right)$, for every $i$. If $I$ is both $\Sigma$ and $\Delta$-invariant ideal, we say that $I$ is $(\Sigma, \Delta)$-invariant. 
Proposition 7 ([28], Proposition 2.6). If $A=\sigma(R)\left\langle x_{1}, \ldots, x_{n}\right\rangle$ is a skew $P B W$ extension over $R$ and $I$ is a $(\Sigma, \Delta)$-invariant ideal of $R$, then the following statements hold:

(1) $I A$ is an ideal of $A$ and $I A \cap R=I$. IA is a proper ideal of $A$ if and only if $I$ is proper in $R$. Moreover, if $\sigma_{i}$ is bijective and $\sigma_{i}(I)=I$, for every $i$, then $I A=A I$.

(2) If $I$ is proper and $\sigma_{i}(I)=I$, for every $1 \leqslant i \leqslant n$, then $A / I A$ is a skew $P B W$ extension of $R / I$. In fact, if $I$ is proper and $A$ is bijective, then $A / I A$ is a bijective skew $P B W$ extension of $R / I$.

From Proposition 7 we can see that if $I$ is $(\Sigma, \Delta)$-invariant, then over $\bar{R}:=R / I$ it is induced a systems $(\bar{\Sigma}, \bar{\Delta})$ of endomorphisms $\bar{\Sigma}$ and $\bar{\Sigma}$ derivations $\bar{\Delta}$ defined by $\left.\overline{\sigma_{i}}(r+I)\right)=\sigma_{i}(r)+I$ and $\overline{\delta_{i}}(r+I)=\delta_{i}(r)+I$, for $1 \leqslant i \leqslant n$. We keep the variables $x_{1}, \ldots, x_{n}$ of the extension $A$ to the extension $A / I A$ if no confusion arises. For quotients of skew PBW extensions, we consider the notion of weak $(\Sigma, \Delta)$-symmetric in the natural way following Definition 4.

Our next theorem generalizes [42], Theorem 3.7.

Theorem 5. Let I be an $(\Sigma, \Delta)$-invariant and weak $(\Sigma, \Delta)$-symmetric ideal of $R$. If $I \subseteq \operatorname{nil}(R)$, then $R / I$ is a weak $(\bar{\Sigma}, \bar{\Delta})$-symmetric ring if and only if $R$ is a weak $(\Sigma, \Delta)$-symmetric ring.

Proof. Consider elements $a, b, c \in R$ such that $(a+I)(b+I)(c+I) \in$ $\operatorname{nil}(R / I)$. There exists a positive integer $m$ with $(a b c)^{m} \in I$. Since $I \subseteq$ $\operatorname{nil}(R)$ it follows that $a b c \in \operatorname{nil}(R)$. By assumption, $R$ is weak $(\Sigma, \Delta)$ symmetric, so $a c \sigma_{i}(b), a c \delta_{i}(b) \in \operatorname{nil}(R)$, for $i=1, \ldots, n$. Hence $(a+I)(c+$ $I)\left(\sigma_{i}(b)+I\right),(a+I)(c+I)\left(\delta_{i}(b)+I\right) \in \operatorname{nil}(R / I)$, that is, $(a+I)(c+$ $I) \overline{\sigma_{i}}(b+I),(a+I)(c+I) \overline{\delta_{i}}(b+I) \in \operatorname{nil}(R / I)$. Therefore $R / I$ is weak $(\bar{\Sigma}, \bar{\Delta})$-symmetric.

Conversely, suppose that $R / I$ is a weak $(\Sigma, \Delta)$-symmetric ring. Consider elements $a, b, c \in R$ with $a b c \in \operatorname{nil}(R)$. It is clear that $(a+I)(b+$ $I)(c+I) \in \operatorname{nil}(R / I)$. Since $R / I$ is weak $(\bar{\Sigma}, \bar{\Delta})$-symmetric, we have that $(a+I)(c+I)\left(\sigma_{i}(b)+I\right)=\left(a c \sigma_{i}(b)+I\right),(a+I)(c+I)\left(\delta_{i}(b)+I\right)=$ $\left(a c \delta_{i}(b)+I\right) \in \operatorname{nil}(R / I)$, for $i=1, \ldots, n$. This means that for every $i$ there exist positive integers $p=p(i), q=q(i)$ depending on $i$, such that $\left(a c \sigma_{i}(b)\right)^{p},\left(a c \delta_{i}(b)\right)^{q} \in I$. In this way, $a c \sigma_{i}(b), a c \delta_{i}(b) \in I$ because $I \subseteq \operatorname{nil}(R)$ which shows that $R$ is a weak $(\bar{\Sigma}, \bar{\Delta})$-symmetric ring.

The next theorem extends [42], Theorem 3.9. 
Theorem 6. If $A=\sigma(R)\left\langle x_{1}, \ldots, x_{n}\right\rangle$ is a skew $P B W$ extension over a $(\Sigma, \Delta)$-compatible and reversible ring $R$, then $R$ is a weak $(\Sigma, \Delta)$-symmetric ring if and only if $A$ is a weak $(\bar{\Sigma}, \bar{\Delta})$-symmetric ring, where the sets of injective endomorphisms $\bar{\Sigma}$ and $\bar{\Sigma}$-derivations $\bar{\Delta}$ of $A$ are as in Proposition 5 .

Proof. If $A$ is a weak $(\bar{\Sigma}, \bar{\Delta})$-symmetric ring, then it is clear that $R$ is weak $(\Sigma, \Delta)$-symmetric ring because $\sigma_{i}(R), \delta_{i}(R) \subseteq R$, for every $i=1, \ldots, n$.

Conversely, suppose that $R$ is weak $(\Sigma, \Delta)$-symmetric ring. Consider the elements $f=\sum_{i=0}^{s} a_{i} X_{i}, g=\sum_{j=0}^{t} b_{j} Y_{j}$ and $h=\sum_{k=0}^{l} c_{k} Z_{k}$ of $A$. From Theorem 2 we know that $a_{i} b_{j} c_{k} \in \operatorname{nil}(R)$, for all $i, j, k$, whence $a_{i} c_{k} \sigma_{l}\left(b_{j}\right), a_{i} c_{k} \delta_{l}\left(b_{j}\right) \in \operatorname{nil}(R)$, for $l=1, \ldots, n$, since $R$ is weak $(\Sigma, \Delta)$ symmetric. Again, Theorem 2 implies that $f h \overline{\sigma_{i}}(g), f h \overline{\delta_{i}}(g) \in \operatorname{nil}(A)$, that is, $A$ is a weak $(\Sigma, \Delta)$-symmetric ring.

Corollary 3 ([42], Corollary 3.10). Let $R$ be a reversible ring. Then $R$ is a weak symmetric ring if and only if $R[t]$ is weak symmetric.

\section{Examples}

The importance of the results presented in this paper is appreciated when we can apply them to algebraic structures more general than skew polynomial rings. In this way, our aim in this section is to provide several examples of noncommutative rings which are skew PBW extensions but not skew polynomial rings. Our list of examples is not exhaustive, so another algebraic structures can be found in [11] or [32].

Let us start with universal enveloping algebras of finite-dimensional Lie algebras over fields. If $\mathfrak{g}$ is a finite dimensional Lie algebra over $\mathbb{k}$ with basis $\left\{x_{1}, \ldots, x_{n}\right\}$, then the universal enveloping algebra of $\mathfrak{g}$, denoted by $U(\mathfrak{g})$, is the algebra generated by the indeterminates $x_{1}, \ldots, x_{n}$ subject to the relations $x_{i} r-r x_{i}=0 \in \mathbb{k}$, for every element $r \in \mathbb{k}$, and $x_{i} x_{j}-x_{j} x_{i}=\left[x_{i}, x_{j}\right] \in \mathfrak{g}$, where $\left[x_{i}, x_{j}\right] \subseteq \mathbb{k}+\mathbb{k} x_{1}+\cdots+\mathbb{k} x_{n}$, for all $1 \leqslant i, j \leqslant n$. Since these enveloping algebras are PBW extensions over $\mathbb{k}$ in the sense of Bell and Goodearl [8] (note that these authors presented another examples of enveloping rings related to enveloping universal algebras), of course are skew PBW extensions also over the field $\mathbb{k}$ ([32], section 3.1). As it is well-known, in general, these algebras are not skew polynomial rings even including non-zero trivial derivations, so it is not possible to apply the results obtained by Ouyang and Chen [42] to guarantee the symmetry of these objects. Of course, it is easy to see that this kind of algebras satisfy the assumptions considered in sections 3 
and 4, so we can guarantee its symmetry by using the results obtained in these sections.

Another family of rings which include the universal enveloping algebra $U(\mathfrak{s l}(2, \mathbb{k}))$, the dispin algebra $U(\mathfrak{o s p}(1,2))$ and the Woronowicz's algebra $W_{\nu}(\mathfrak{s l}(2, \mathbb{k}))$, is called the family of 3-dimensional skew polynomial algebras. These algebras were introduced by Bell and Smith [9] and are very important in noncommutative algebraic geometry (Rosenberg [51]). We recall its definition and classification.

Definition 6 ([51], Definition C4.3). A 3-dimensional skew polynomial algebra $A$ is a $\mathbb{k}$-algebra generated by the variables $x, y, z$ restricted to relations $y z-\alpha z y=\lambda, z x-\beta x z=\mu$, and $x y-\gamma y x=\nu$, such that

1) $\lambda, \mu, \nu \in \mathbb{k}+\mathbb{k} x+\mathbb{k} y+\mathbb{k} z$, and $\alpha, \beta, \gamma \in \mathbb{k} \backslash\{0\}$;

2) Standard monomials $\left\{x^{i} y^{j} z^{l} \mid i, j, l \geqslant 0\right\}$ are a $\mathbb{k}$-basis of the algebra.

From Definition 6, it is clear that these algebras are skew PBW extensions over the field $\mathbb{k}$, that is, $A \cong \sigma(\mathbb{k})\langle x, y, z\rangle$.

Proposition 8 ([51], Theorem C.4.3.1). If $A$ is a 3-dimensional skew polynomial algebra, then $A$ is one of the following algebras:

(a) if $|\{\alpha, \beta, \gamma\}|=3$, then $A$ is defined by the relations $y z-\alpha z y=$ $0, z x-\beta x z=0, x y-\gamma y x=0$.

(b) if $|\{\alpha, \beta, \gamma\}|=2$ and $\beta \neq \alpha=\gamma=1$, then $A$ is one of the following algebras:

(i) $y z-z y=z, \quad z x-\beta x z=y, \quad x y-y x=x$;

(ii) $y z-z y=z, \quad z x-\beta x z=b, \quad x y-y x=x$;

(iii) $y z-z y=0, \quad z x-\beta x z=y, \quad x y-y x=0$;

(iv) $y z-z y=0, \quad z x-\beta x z=b, \quad x y-y x=0$;

(v) $y z-z y=a z, \quad z x-\beta x z=0, \quad x y-y x=x$;

(vi) $y z-z y=z, \quad z x-\beta x z=0, \quad x y-y x=0$,

where $a, b$ are any elements of $\mathbb{k}$. All nonzero values of $b$ give isomorphic algebras.

(c) If $|\{\alpha, \beta, \gamma\}|=2$ and $\beta \neq \alpha=\gamma \neq 1$, then $A$ is one of the following algebras:

(i) $y z-\alpha z y=0, \quad z x-\beta x z=y+b, \quad x y-\alpha y x=0$;

(ii) $y z-\alpha z y=0, \quad z x-\beta x z=b, \quad x y-\alpha y x=0$.

In this case, $b$ is an arbitrary element of $\mathbb{k}$. Again, any nonzero values of $b$ give isomorphic algebras.

(d) If $\alpha=\beta=\gamma \neq 1$, then $A$ is the algebra defined by the relations $y z-\alpha z y=a_{1} x+b_{1}, z x-\alpha x z=a_{2} y+b_{2}, x y-\alpha y x=a_{3} z+b_{3}$. If $a_{i}=0(i=1,2,3)$, then all nonzero values of $b_{i}$ give isomorphic algebras. 
(e) If $\alpha=\beta=\gamma=1$, then $A$ is isomorphic to one of the following algebras:

(i) $y z-z y=x, \quad z x-x z=y, \quad x y-y x=z$;

(ii) $y z-z y=0, \quad z x-x z=0, \quad x y-y x=z$;

(iii) $y z-z y=0, \quad z x-x z=0, \quad x y-y x=b$;

(iv) $y z-z y=-y, \quad z x-x z=x+y, \quad x y-y x=0$;

(v) $y z-z y=a z, \quad z x-x z=z, \quad x y-y x=0$;

Parameters $a, b \in \mathbb{k}$ are arbitrary, and all nonzero values of $b$ generate isomorphic algebras.

As we said before, every 3-dimensional skew polynomial algebra is a skew PBW extension. Nevertheless, some of these algebras cannot be expressed as skew polynomial rings even in the case of non-trivial derivations. One of the illustrative examples of this fact is the Dispin algebra $U(\operatorname{osp}(1,2))$, which is the enveloping algebra of the Lie superalgebra $\mathfrak{o s p}(1,2)$ ([51], Definition C4.1). By definition, Dispin algebra is generated by the indeterminates $x, y, z$ over a field $\mathbb{k}$ satisfying the relations $y z-z y=$ $z, z x+x z=y$ and $x y-y x=x$ (the algebra (b)(i) above with $\beta=-1$ ). As can be seen, it appears that this algebra is not a skew polynomial ring of automorphism type but it is a skew PBW extension. Hence, the symmetry of this algebra follows from the results presented in sections 3 and 4.

The quantum algebra $U_{q}^{\prime}\left(\mathfrak{s o}_{3}\right)$ is another example of a skew PBW extension which cannot be expressed as skew polynomial ring of automorphism type. This algebra was introduced by Gavrilik and Klimik [12] and is a nonstandard $q$-deformation of the universal enveloping algebra $U\left(\mathfrak{s o}_{3}\right)$ of the Lie algebra $\mathfrak{s o}_{3}$. More exactly, given $q \in \mathbb{k} \backslash\{0\}, U_{q}^{\prime}\left(\mathfrak{s o}_{3}\right)$ is defined as the algebra generated by the intederminates $I_{1}, I_{2}$, and $I_{3}$ over $\mathbb{k}$ satisfying the relations $I_{2} I_{1}-q I_{1} I_{2}=-q^{\frac{1}{2}} I_{3}, I_{3} I_{1}-q^{-1} I_{1} I_{3}=q^{-\frac{1}{2}} I_{2}$, and $I_{3} I_{2}-q I_{2} I_{3}=-q^{-\frac{1}{2}} I_{1}$. From its definition, one can think that this algebra is not a skew polynomial of automorphism type since the commutation rule of two indeterminates involves the third ring but one can check that $U_{q}^{\prime}\left(\mathfrak{s o}_{3}\right) \cong \sigma(\mathbb{k})\left\langle I_{1}, I_{2}, I_{3}\right\rangle$.

Finally, diffusion algebras arose in physics as a possible way to understand a large class of 1-dimensional stochastic process, see Isaev et al. [21]. A diffusion algebra $A$ with parameters $a_{i j} \in \mathbb{C} \backslash\{0\}, 1 \leqslant i, j \leqslant n$ is an algebra over $\mathbb{C}$ generated by variables $x_{1}, \ldots, x_{n}$ subject to relations $a_{i j} x_{i} x_{j}-b_{i j} x_{j} x_{i}=r_{j} x_{i}-r_{i} x_{j}$, whenever $i<j, b_{i j}, r_{i} \in \mathbb{C}$, for all $i<j$, such that the indeterminates $x$ 's form a $\mathbb{C}$-basis of the algebra $A$. In the applications to physics the parameters $a_{i j}$ are strictly positive reals and 
the parameters $b_{i j}$ are positive reals as they are unnormalised measures of probability. As we can see, these algebras are not skew polynomial rings over $\mathbb{C}\left[x_{1}, \ldots, x_{n}\right]$ but are skew PBW extensions over this ring satisfying the conditions imposed in theorems presented in sections 3 and 4, so we can assert their symmetry.

\section{References}

[1] J. P. Acosta, O. Lezama. Universal property of skew PBW extensions, Algebra Discrete Math. 20 (2015), no. 1, 1-12.

[2] D. D. Anderson, V. Camillo. Armendariz rings and Gaussian rings, Comm. Algebra 26 (1998), no. 7, 2265-2272.

[3] S. Annin S. Associated primes over Ore extension rings, J. Algebra Appl. 3 (2004), no. 2, 193-205.

[4] V. A. Artamonov. Derivations of skew PBW extensions, Commun. Math. Stat. 3 (2015), no. 4, 449-457.

[5] V. A. Artamonov, O. Lezama, W. Fajardo. Extended modules and Ore extensions, Commun. Math. Stat. 4 (2016), no. 2, 189-202.

[6] J. Apel. Gröbnerbasen in nichtkommutativen Algebren und ihre Anwendung. (Gröbner bases in noncommutative algebras and their applications). Leipzig: KarlMarx-Univ. (1988).

[7] H. E. Bell. Near-rings in which each element is a power of itself, Bull. Aust. Math. Soc. 2 (1970), no. 3, 363-368.

[8] A. D. Bell, K. Goodearl. Uniform rank over differential operator rings and PoincaréBirkhoff-Witt extensions, Pacific J. Math. 131 (1988), no. 11, 13-37.

[9] A. D. Bell, S. P. Smith. Some 3-dimensional skew polynomial ring. University of Wisconsin, Milwaukee, preprint (1990).

[10] J. Bueso, J. Gómez-Torrecillas, A. Verschoren. Algorithmic Methods in Noncommutative Algebra. Applications to Quantum Groups. Dordrecht, Kluwer (2003).

[11] W. Fajardo, C. Gallego, O. Lezama, A. Reyes, H. Suárez, H. Venegas. Skew PBW Extensions. Ring and Module-theoretic Properties, Matrix and Gröbner Methods, and Applications. Algebra and Applications. Springer Cham (2020).

[12] A. M. Gavrilik, A. U. Klimik, $q$-Deformed orthogonal and pseudo-orthogonal algebras and their representations. Lett. Math. Phys. 21 (1991), no. 3, 215-220.

[13] C. Gallego, O. Lezama. Gröbner bases for ideals of $\sigma$-PBW extensions, Comm. Algebra 39 (2011), no. 1, 50-75.

[14] C. Gallego, O. Lezama. Matrix approach to noncommutative stably free modules and Hermite rings, Algebra Discrete Math. 18 (2014), no. 1, 109-137.

[15] M. Hamidizadeh, E. Hashemi, A. Reyes. A classification of ring elements in skew PBW extensions over compatible rings, Int. Electron. J. Algebra 28 (2020), 75-97.

[16] E. Hashemi, A. Moussavi, H. Haj Seyyed Javadi. Polynomial Ore extensions of Baer and p.p.-rings, Bull. Iranian Math. Soc. 29 (2003), no. 2, 65-86. 
[17] E. Hashemi, K. Khalilnezhad, A. Alhevaz, A. ( $\Sigma, \Delta)$-Compatible Skew PBW Extension Ring, Kyungpook Math. J. 57 (2017), no. 3, 401-417.

[18] E. Hashemi, K. Khalilnezhad, A. Alhevaz. Extensions of rings over 2-primal rings, Matematiche (Catania) $\mathbf{7 4}$ (2019), no. 1, 141-162.

[19] E. Hashemi, K. Khalilnezhad, M. Ghadiri. Baer and quasi-Baer properties of skew PBW extensions, J. Algebr. Syst. 7 (2019), no. 1, 1-24.

[20] C. Huh, C. Lee, A. Smoktunowicz. Armendariz rings and semicommutative rings, Comm. Algebra 30 (2002), no. 2, 751-761.

[21] A. P. Isaev, P. N. Pyatov, V. Rittenberg. Diffusion algebras, J. Phys. A. 34 (2001), no. $29,5815-5834$.

[22] H. Jiménez, O. Lezama. Gröbner bases of modules over $\sigma$-PBW extensions, Acta Math. Acad. Paedagog. Nyházi. (N.S.) 32 (2016), 39-66.

[23] A. Kandri-Rody, V. Weispfenning. Non-commutative Gröbner Bases in Algebras of Solvable Type, J. Symbolic Computation 9 (1990), no. 1, 1-26.

[24] N. Kim, Y. Lee. Extensions of reversible rings. J. Pure Appl. Algebra, 185 (2003), no. 1-3, 207-223.

[25] J. Krempa. Some examples of reduced rings, Algebra Colloq. 3 (1996), no. 4, 289-300.

[26] J. Lambek. On the representation of modules by sheaves of factor modules, Canad. Math. Bull. 14 (1971), 359-368.

[27] O. Lezama. Computation of point modules of finitely semi-graded rings, Comm. Algebra 48 (2020), no. 2, 866-878.

[28] O. Lezama, J. P. Acosta, A. Reyes. Prime ideals of skew PBW extensions, Rev. Un. Mat. Argentina 56 (2015), no. 2, 39-55.

[29] O. Lezama, C. Gallego. $d$-Hermite rings and skew PBW extensions, São Paulo J. Math. Sci. 10 (2016), no. 1, 60-72.

[30] O. Lezama, J. Gómez. Koszulity and point modules of finitely semi-graded rings and algebras, Symmetry 11 (2019), no. 7, 1-22.

[31] O. Lezama, E. Latorre. Non-commutative algebraic geometry of semi-graded rings, Internat. J. Algebra Comput. 27 (2017), no. 4, 361-389.

[32] O. Lezama, A. Reyes. Some Homological Properties of Skew PBW Extensions, Comm. Algebra 42 (2014), no. 3, 1200-1230.

[33] O. Lezama, H. Venegas. Center of skew PBW extensions, Internat. J. Algebra Comput. 30 (2020), no. 8, 1625-1650.

[34] Z. Liu, R. Zhao, On weak Armendariz rings, Comm. Algebra 37 (2006), no. 7, 2607-2616.

[35] M. Louzari, A. Reyes. Minimal prime ideals of skew PBW extensions over 2-primal compatible rings, Rev. Colombiana Mat. 54 (2020), no. 1, 39-63.

[36] G. Marks. A taxonomy of 2-primal rings, J. Algebra 266 (2003), no. 2, 494-520.

[37] J. C. McConnell, J. C. Robson. Noncommutative Noetherian Rings, Graduate Studies in Mathematics, AMS (2001). 
[38] A. Moussavi, E. Hashemi. On $(\alpha, \delta)$-skew Armendariz rings, J. Korean Math. Soc. 42 (2005), no. 2, 353-363.

[39] A. Niño, M. C. Ramírez, A. Reyes. Associated prime ideals over skew PBW extensions, Comm. Algebra 48 (2020), no. 12, 5038-5055.

[40] A. Niño, A. Reyes. Some remarks about minimal prime ideals of skew PBW extensions, Algebra Discrete Math. 30 (2020), no. 2, 207-229.

[41] O. Ore. Theory of Non-Commutative Polynomials, Ann. of Math. (2) 34 (1933), no. $3,480-508$.

[42] L. Ouyang, H. Chen H. On weak symmetric rings, Comm. Algebra 38 (2010), 697-713.

[43] M. B. Rege, S. Chhawchharia. Armendariz rings, Proc. Japan Acad. Ser. A Math. Sci. 73 (1997), no. 1, 14-17.

[44] A. Reyes. Skew PBW extensions of Baer, quasi-Baer, p.p. and p.q.-rings, Rev. Integr. Temas Mat. 33 (2015), no. 2, 173-189.

[45] A. Reyes, C. Rodríguez. The McCoy condition on Skew Poincaré-Birkhoff-Witt Extensions, Commun. Math. Stat. 9 (2021), no. 1, 1-21.

[46] A. Reyes, H. Suárez. PBW bases for some 3-dimensional skew polynomial algebras, Far East J. Math. Sci. 101 (2017), no. 6, 1207-1228.

[47] A. Reyes, H. Suárez. $\sigma$-PBW Extensions of Skew Armendariz Rings, Adv. Appl. Clifford Algebr. 27 (2017), no. 4, 3197-3224.

[48] A. Reyes, H. Suárez. A notion of compatibility for Armendariz and Baer properties over skew PBW extensions, Rev. Un. Mat. Argentina 59 (2018), no. 1, 157-178.

[49] A. Reyes, H. Suárez. Skew Poincaré-Birkhoff-Witt extensions over weak zip rings, Beitr. Algebra Geom. 60 (2019), no. 2, 190-216.

[50] A. Reyes, H. Suárez. Radicals and Köthe's conjecture for skew PBW extensions, Commun. Math. Stat. 9 (2021), no. 2, 119-138.

[51] A. Rosenberg. Non-commutative Algebraic Geometry and Representations of Quantized Algebras. Mathematics and Its Applications, Vol. 330. Kluwer Academic Publishers (1995).

[52] H. Suárez. Koszulity for graded skew PBW extensions, Comm. Algebra 45 (2017), no. 10, 4569-4580.

[53] H. Suárez, O. Lezama and A. Reyes. Calabi-Yau property for graded skew PBW extensions, Rev. Colombiana Mat. 51 (2017), no. 2, 231-238.

[54] A. B. Tumwesigye, J. Richter, S. Silvestrov. Centralizers in PBW Extensions. In: Silvestrov S., Malyarenko A., Rancić M. (eds) Algebraic Structures and Applications. SPAS 2017. Springer Proceedings in Mathematics \& Statistics, Vol. 317, Springer, Cham (2020).

[55] B. A. Zambrano. Poisson brackets on some skew PBW extensions, Algebra Discrete Math. 29 (2020), no. 2, 277-302. 
CONTACT INFORMATION

Armando Reyes

Department of Mathematics

Faculty of Science

Universidad Nacional de Colombia - Sede Bogotá

E-Mail(s): mareyesv@unal .edu.co

Héctor Suárez

School of Mathematics and Statistics Faculty of Science

Universidad Pedagógica y Tecnológica de Colombia, Tunja

E-Mail(s): hector.suarez@uptc.edu.co

Received by the editors: 14.02.2021. 